Pacific

Journal of

Mathematics

TOPOLOGY OF THE MODULI OF REPRESENTATIONS WITH BOREL MOLD

KAZUNORI NAKAMOTO AND TAKESHI TORI

Volume $213 \quad$ No. 2

February 2004 


\title{
TOPOLOGY OF THE MODULI OF REPRESENTATIONS WITH BOREL MOLD
}

\author{
KAZUnORI NAKAMOTO AND TAKESHI TORII
}

\begin{abstract}
We give descriptions of the moduli of representations with Borel mold for free monoids as fibre bundles over the configuration spaces. By using the associated Serre spectral sequences, we study the cohomology rings of the moduli. Also we calculate the virtual Hodge polynomials of them.
\end{abstract}

\section{Introduction.}

A representation for a group or a monoid is called a representation with Borel mold if it can be normalized to a representation in upper triangular matrices whose image of the group or monoid generates the algebra of upper triangular matrices. In [Na2] the moduli of representations with Borel mold has been constructed for each group or monoid. The moduli of representations with Borel mold has simpler structure than the moduli of absolutely irreducible representations constructed in [Na1]. In the present paper, for the free monoid case we describe the moduli of representations with Borel mold explicitly, and calculate its cohomology ring.

The moduli of representations with Borel mold has a fibre bundle structure over the configuration space of the affine space, and hence its cohomology ring can be calculated. We also calculate the virtual Hodge polynomial of the moduli of representations with Borel mold, which will be used for calculating the virtual Poincaré polynomial of the moduli of absolutely irreducible representations of degree 2 for the free monoid case in [Na3]. By calculating the cohomology ring of the moduli, we can consider characteristic classes for representations with Borel mold on a scheme. The construction of characteristic classes and its application will be presented in other papers.

By global representation theory we understand theory of representations on (arbitrary) schemes. The global representation theory is geometric rather than the local representation theory, that is, the representation theory over fields or local rings. For example, each representation of degree $n$ with Borel mold for a group (or a monoid) $\Gamma$ on a scheme $X$ has a unique $\Gamma$-invariant complete flag of $\mathcal{O}_{X}^{n}$ (see [Na2]). The $\Gamma$-invariant complete flag is not always trivial on $X$, although if $X$ is the spectrum of a field or a local ring, then the flag is trivial. Non-triviality of the $\Gamma$-invariant flag is an interesting feature of the theory of representations over schemes. 
As above, the global representation theory has a geometric aspect. For developing the global representation theory, in particular, the theory of representations with Borel mold, we need to consider topology of the moduli of representations with Borel mold. If we intend to construct characteristic classes of representations with Borel mold on schemes (which seems to be an important tool for the global representation theory in the future), then we have to calculate the (ordinary) cohomology of the moduli. That is our main motivation. In this article, we deal with only the free monoid case. The free monoid case is a fundamental case for considering the moduli of representations with Borel mold.

Let us go into details on our main results. There is a 1-1 correspondence between the representations with Borel mold of the free monoid of rank $m$ and the $m$-matrices of size $n \times n$ which generate the algebra of upper triangular matrices. We study the condition for $m$-upper triangular matrices to generate the algebra and it gives us a description of the moduli $\mathrm{Ch}_{n}(m)_{B}$ of representations with Borel mold for the free monoid of rank $m$ as a fibre bundle over the configuration space $F_{n}\left(\mathbb{A}_{\mathbb{Z}}^{m}\right)$ of the affine space $\mathbb{A}_{\mathbb{Z}}^{m}$.

Theorem 1.1 (Proposition 3.8). The moduli $\mathrm{Ch}_{n}(m)_{B}$ of representations with Borel mold is a fibre bundle over the configuration space $F_{n}\left(\mathbb{A}_{\mathbb{Z}}^{m}\right)$ of the affine space $\mathbb{A}_{\mathbb{Z}}^{m}$ with fibre $\left(\mathbb{P}_{\mathbb{Z}}^{m-2}\right)^{n-1} \times\left(\mathbb{A}_{\mathbb{Z}}^{m-1}\right)^{(n-2)(n-1) / 2}$ with respect to Zariski topology.

Thereby we can calculate the cohomology rings of $\mathrm{Ch}_{n}(m)_{B}$ and related varieties which are regarded as algebraic schemes over $\mathbb{C}$ by tensoring with $\mathbb{C}$. The description of $\mathrm{Ch}_{n}(m)_{B}$ as a fibre bundle gives us the Serre spectral sequence converging to the cohomology ring of $\mathrm{Ch}_{n}(m)_{B}$. The structure of the cohomology ring of the configuration space $F_{n}\left(\mathbb{C}^{m}\right)$ is well-known (cf. [Co1] and [Co2]). Then it is easy to show that the spectral sequence collapses from the $E_{2}$-term.

Theorem 1.2 (Theorem 5.2). The cohomology ring of $\mathrm{Ch}_{n}(m)_{B}$ is given by

$$
H^{*}\left(\mathrm{Ch}_{n}(m)_{B}\right) \cong H^{*}\left(F_{n}\left(\mathbb{C}^{m}\right)\right) \otimes \mathbb{Z}\left[t_{1}, \ldots, t_{n-1}\right] /\left(t_{1}^{m-1}, \ldots, t_{n-1}^{m-1}\right),
$$

where the degree $\left|t_{j}\right|=2$ for $1 \leq j \leq n-1$.

From Deligne's mixed Hodge theory ([De1] and [De2]), we have an invariant of algebraic varieties over $\mathbb{C}$ called the virtual Hodge polynomial which is a generalization of the Hodge polynomial for smooth projective varieties over $\mathbb{C}$. The virtual Hodge polynomial has a good property for fibre bundles with respect to Zariski topology. We calculate the virtual Hodge polynomials of $\mathrm{Ch}_{n}(m)_{B}$ and related varieties. 
Theorem 1.3 (Proposition 7.8). The virtual Hodge polynomial of the moduli $\mathrm{Ch}_{n}(m)_{B}$ is given by

$$
H\left(\mathrm{Ch}_{n}(m)_{B}\right)=\frac{\left(z^{m-1}-1\right)^{n-1}}{(z-1)^{n-1}} z^{(m-1)(n-2)(n-1) / 2} \prod_{k=0}^{n-1}\left(z^{m}-k\right) .
$$

The organization of this paper is as follows: In $\S 2$ we review the moduli of representations with Borel mold. In $\S 3$ we give descriptions of the moduli schemes $\mathrm{B}_{n}(m)_{B}, \mathrm{Ch}_{n}(m)_{B}$ and $\operatorname{Rep}_{n}(m)_{B}$. We show that $\mathrm{B}_{n}(m)_{B}$ and $\mathrm{Ch}_{n}(m)_{B}$ are fibre bundles over the configuration space $F_{n}\left(\mathbb{A}_{\mathbb{Z}}^{m}\right)$, and $\operatorname{Rep}_{n}(m)_{B}$ is a fibre bundle over the flag scheme $\operatorname{Flag}\left(\mathbb{A}_{\mathbb{Z}}^{n}\right)$ with respect to Zariski topology. From the descriptions as fibre bundles, we study the associated Serre spectral sequences and calculate the cohomology rings of $\mathrm{B}_{n}(m)_{B}, \mathrm{Ch}_{n}(m)_{B}$ and $\operatorname{Rep}_{n}(m)_{B}$ in $\S \S 4,5,6$. In $\S 7$ we calculate the virtual Hodge polynomials of $\mathrm{B}_{n}(m)_{B}, \mathrm{Ch}_{n}(m)_{B}$ and $\operatorname{Rep}_{n}(m)_{B}$. In $\S 8$ we define $\mathrm{B}_{n}(\infty)_{B}, \mathrm{Ch}_{n}(\infty)_{B}$ and $\operatorname{Rep}_{n}(\infty)_{B}$ to be the homotopy direct limits of natural inclusions respectively, and study the homotopy types and the cohomology rings of them.

\section{Survey: The moduli of representations with Borel mold.}

In this section, we make a survey of the moduli of representations with Borel mold. We use [Na2] as our main reference.

2.1. Representations with Borel mold. Let $\Gamma$ be a group or a monoid. Let $X$ be a scheme. By a representation of degree $n$ for $\Gamma$ on $X$ we understand a group (resp. monoid) homomorphism $\Gamma \rightarrow \mathrm{GL}_{n}\left(\Gamma\left(X, \mathcal{O}_{X}\right)\right)$ (resp. $\Gamma \rightarrow \mathrm{M}_{n}\left(\Gamma\left(X, \mathcal{O}_{X}\right)\right)$ ).

For two representations $\rho, \rho^{\prime}$ of degree $n$ for $\Gamma$ on $X$, we say that $\rho$ and $\rho^{\prime}$ are equivalent (or $\left.\rho \sim \rho^{\prime}\right)$ if there exists a $\Gamma\left(X, \mathcal{O}_{X}\right)$-algebra isomorphism $\sigma: \mathrm{M}_{n}\left(\Gamma\left(X, \mathcal{O}_{X}\right)\right) \rightarrow \mathrm{M}_{n}\left(\Gamma\left(X, \mathcal{O}_{X}\right)\right)$ such that $\sigma(\rho(\gamma))=\rho^{\prime}(\gamma)$ for each $\gamma \in \Gamma$.

By a mold of degree $n$ on a scheme $X$ we understand a subsheaf of $\mathcal{O}_{X^{-}}$ algebras of $\mathrm{M}_{n}\left(\mathcal{O}_{X}\right)$ which is also a subbundle of $\mathrm{M}_{n}\left(\mathcal{O}_{X}\right)$. By two molds $\mathcal{A}$ and $\mathcal{B}$ of degree $n$ on $X$, we say that $\mathcal{A}$ and $\mathcal{B}$ are locally equivalent if there exist an open covering $X=\cup_{i \in I} U_{i}$ and $P_{i} \in \operatorname{GL}_{n}\left(\Gamma\left(U_{i}, \mathcal{O}_{X}\right)\right)$ such that $P_{i}^{-1}\left(\left.\mathcal{A}\right|_{U_{i}}\right) P_{i}=\left.\mathcal{B}\right|_{U_{i}}$. We define the mold $\mathcal{B}_{n}$ on Spec $\mathbb{Z}$ by $\mathcal{B}_{n}:=\left\{\left(b_{i j}\right) \in \mathrm{M}_{n}(\mathbb{Z}) \mid b_{i j}=0\right.$ for each $\left.i>j\right\}$. For a mold $\mathcal{A}$ of degree $n$ on $X$ we say that $\mathcal{A}$ is a Borel mold of degree $n$ if $\mathcal{A}$ and $\mathcal{B}_{n} \otimes_{\mathbb{Z}} \mathcal{O}_{X}$ are locally equivalent.

Under the above preparations, we introduce the notion of representations with Borel mold.

Definition 2.1. For a representation $\rho$ of degree $n$ for a group (or a monoid) $\Gamma$ on a scheme $X$ we say that $\rho$ is a representation with Borel mold if the subsheaf $\mathcal{O}_{X}[\rho(\Gamma)]$ of $\mathrm{M}_{n}\left(\mathcal{O}_{X}\right)$ generated by $\rho(\Gamma)$ is a Borel mold. 
2.2. Review of the moduli of representations with Borel mold. Let $\Gamma$ be a group or a monoid. The following functor is representable by an affine scheme:

$$
\begin{aligned}
\operatorname{Rep}_{n}(\Gamma):(\mathbf{S c h})^{o} & \rightarrow(\text { Sets }) \\
X & \mapsto \text { representations of degree } n \text { for } \Gamma \text { on } X\} .
\end{aligned}
$$

The affine scheme $\operatorname{Rep}_{n}(\Gamma)$ is called the representation variety of degree $n$ for $\Gamma$.

Definition 2.2. We define the locally closed subscheme $\operatorname{Rep}_{n}(\Gamma)_{B}$ of the affine scheme $\operatorname{Rep}_{n}(\Gamma)$ which represents the functor

$$
\left.\begin{array}{rl}
\operatorname{Rep}_{n}(\Gamma)_{B}:(\mathbf{S c h})^{o} & \rightarrow(\text { Sets }) \\
X & \mapsto\left\{\rho \in \operatorname{Rep}_{n}(\Gamma)(X) \mid \rho:\right. \text { representation } \\
\text { with Borel mold }
\end{array}\right\} .
$$

Definition 2.3. We define the closed subscheme $\mathrm{B}_{n}(\Gamma)$ of $\operatorname{Rep}_{n}(\Gamma)$ which represents the functor

$$
\begin{aligned}
\mathrm{B}_{n}(\Gamma):(\mathbf{S c h})^{o} & \rightarrow\left\{\begin{array}{l|l}
\rho \in \operatorname{Rep}_{n}(\Gamma)(X) & \begin{array}{c}
\text { the }(i, j) \text {-entry of } \\
\rho(\gamma)=0 \text { for each } i>j \\
\text { and for each } \gamma \in \Gamma
\end{array}
\end{array}\right\} .
\end{aligned}
$$

We also define the open subscheme $\mathrm{B}_{n}(\Gamma)_{B}$ of $\mathrm{B}_{n}(\Gamma)$ by $\mathrm{B}_{n}(\Gamma)_{B}:=\mathrm{B}_{n}(\Gamma) \cap$ $\operatorname{Rep}_{n}(\Gamma)_{B}$.

The group scheme $\mathrm{PGL}_{n}$ acts on the schemes $\operatorname{Rep}_{n}(\Gamma)$ and $\operatorname{Rep}_{n}(\Gamma)_{B}$ by $\rho \mapsto P^{-1} \rho P$. Let $\mathrm{B}_{n}$ be the closed subgroup scheme of $\mathrm{PGL}_{n}$ defined by $\mathrm{B}_{n}:=\left\{\left(b_{i j}\right) \in \mathrm{PGL}_{n} \mid b_{i j}=0\right.$ for each $\left.i>j\right\}$. The group scheme $\mathrm{B}_{n}$ acts on the schemes $\mathrm{B}_{n}(\Gamma)$ and $\mathrm{B}_{n}(\Gamma)_{B}$ by $\rho \mapsto b \rho b^{-1}$.

We define two group actions on $\mathrm{B}_{n}(\Gamma)_{B} \times \mathrm{PGL}_{n}$ : One is the action of $\mathrm{PGL}_{n}$ defined by $(\rho, P) \mapsto(\rho, P Q)$, and the other is one of $\mathrm{B}_{n}$ defined by $(\rho, P) \mapsto\left(b \rho b^{-1}, b P\right)$. Defining the morphism $\mathrm{B}_{n}(\Gamma)_{B} \times \mathrm{PGL}_{n} \rightarrow \operatorname{Rep}_{n}(\Gamma)_{B}$ by $(\rho, P) \mapsto P^{-1} \rho P$, we obtain the following diagram which is a fibre product:

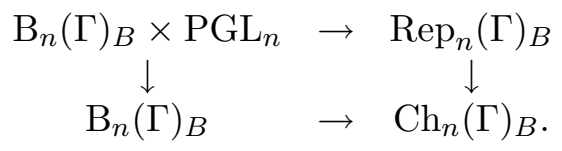

We denote the universal geometric quotient $\mathrm{B}_{n}(\Gamma)_{B} / \mathrm{B}_{n}=\operatorname{Rep}_{n}(\Gamma)_{B} / \mathrm{PGL}_{n}$ by $\mathrm{Ch}_{n}(\Gamma)_{B}$ (the existence of the universal geometric quotient has been proved in [Na2]). The morphism $\mathrm{B}_{n}(\Gamma)_{B} \times \mathrm{PGL}_{n} \rightarrow \mathrm{B}_{n}(\Gamma)_{B}$ is the first projection. The two down arrows are $\mathrm{PGL}_{n}$-principal fibre bundles, and the two right arrows are $\mathrm{B}_{n}$-principal fibre bundles.

Under the above situation, we have the following theorem: 
Theorem $2.4([\mathrm{Na2}])$. The scheme $\mathrm{Ch}_{n}(\Gamma)_{B}$ represents the sheafification of the following functor with respect to Zariski topology:

$$
\begin{aligned}
\mathcal{E}_{q} \mathcal{B}_{n}(\Gamma):(\mathbf{S c h})^{o} & \rightarrow(\text { Sets }) \\
X & \mapsto \operatorname{Rep}_{n}(\Gamma)_{B}(X) / \sim .
\end{aligned}
$$

In other words, the scheme $\mathrm{Ch}_{n}(\Gamma)_{B}$ is the moduli of representations with Borel mold.

By introducing the following notation, we end this section:

Notation 2.5. Let $\Upsilon_{m}$ be the free monoid of rank $m$. For $\operatorname{Rep}_{n}\left(\Upsilon_{m}\right)_{B}$, $\mathrm{B}_{n}\left(\Upsilon_{m}\right)_{B}, \mathrm{Ch}_{n}\left(\Upsilon_{m}\right)_{B}$, we also write $\operatorname{Rep}_{n}(m)_{B}, \mathrm{~B}_{n}(m)_{B}, \mathrm{Ch}_{n}(m)_{B}$, respectively. These are schemes over $\mathbb{Z}$, however in $\S 4-\S 8$ we use these notations for $\operatorname{Rep}_{n}(m)_{B} \otimes_{\mathbb{Z}} \mathbb{C}, \mathrm{B}_{n}(m)_{B} \otimes_{\mathbb{Z}} \mathbb{C}$, and $\operatorname{Ch}_{n}(m)_{B} \otimes_{\mathbb{Z}} \mathbb{C}$, respectively.

\section{Description of the moduli.}

In this section, we describe the moduli of representations with Borel mold of degree $n$ for free monoids by using the configuration spaces. Considering the 1-1 correspondence between representations of the free monoid $\Upsilon_{m}$ and $m$ matrices of size $n \times n$, we see that $\mathrm{B}_{n}(m)_{B}$ is isomorphic to

$$
\left\{\begin{array}{l|l}
\left(A_{1}, \ldots, A_{m}\right) \mid \begin{array}{c}
A_{1}, \ldots, A_{m} \text { generate the algebra } \\
\text { of upper triangular matrices }
\end{array}
\end{array}\right\} .
$$

Hence we will investigate the latter space.

3.1. Preliminaries. In this subsection, we study the condition that $m$ upper triangular matrices generate the algebra of upper triangular matrices.

Let $k$ be a field. We define the $k$-algebra $\mathcal{B}_{n}(k)$ by

$$
\mathcal{B}_{n}(k):=\left\{\left(a_{i j}\right) \in \mathrm{M}_{n}(k) \mid a_{i j}=0 \text { for each } i>j\right\} .
$$

For $n \geq 2$ and $1 \leq i \neq j \leq n$, we define the $k$-linear map

$$
p_{i j}: \begin{array}{ccc}
\mathcal{B}_{n}(k) & \rightarrow & \mathcal{B}_{2}(k) \\
\left(a_{s t}\right)_{1 \leq s, t \leq n} & \mapsto\left(a_{s t}\right)_{s, t=i, j} .
\end{array}
$$

We also define the $k$-algebra homomorphism $\phi_{n}$ by

$$
\begin{aligned}
& \phi_{n}: \mathcal{B}_{n}(k) \quad \rightarrow \quad k^{n} \\
& \left(a_{s t}\right)_{1 \leq s, t \leq n} \mapsto\left(a_{11}, a_{22}, \ldots, a_{n n}\right) .
\end{aligned}
$$

Lemma 3.1. For a $k$-subalgebra $\mathcal{A} \subseteq \mathcal{B}_{2}(k)$, it is equal to $\mathcal{B}_{2}(k)$ if and only if $\mathcal{A}$ is a non-commutative algebra.

Proof. Easy.

Lemma 3.2. For a $k$-subalgebra $\mathcal{A} \subseteq \mathcal{B}_{n}(k)$ with $n \geq 3, \mathcal{A}=\mathcal{B}_{n}(k)$ if and only if $\left.\phi_{n}\right|_{\mathcal{A}}$ is surjective and $\left.p_{i, i+1}\right|_{\mathcal{A}}: \mathcal{A} \rightarrow \mathcal{B}_{2}(k)$ is surjective for each $1 \leq i \leq n-1$. 
Proof. The "only if" part is obvious. Let us show the "if" part. Put $J:=$ $\operatorname{Ker}\left(\left.\phi_{n}\right|_{\mathcal{A}}\right)=\left\{\left(a_{i j}\right) \in \mathcal{A} \mid a_{11}=a_{22}=\cdots=a_{n n}=0\right\}$. First we claim that for each $1 \leq i \leq n-1$ there exists $P_{i}=\left(a_{s t}^{i}\right) \in J$ such that $a_{i, i+1}^{i}=1$ and $a_{j, j+1}^{i}=0$ for each $j(\neq i)$. Since $\left.p_{i, i+1}\right|_{\mathcal{A}}$ is surjective, there exists $P^{\prime}=\left(b_{s t}\right) \in \mathcal{A}$ such that $b_{i i}=b_{i+1, i+1}=0$ and $b_{i, i+1}=1$. By the surjectivity of $\left.\phi_{n}\right|_{\mathcal{A}}$ we also have $Q:=\left(c_{s t}\right) \in \mathcal{A}$ such that $c_{i i}=1$ and $c_{j j}=0$ for each $j(\neq i)$. Then the matrix $P_{i}:=Q^{2} P^{\prime}$ is what we want.

Next let $P_{i}$ be as above. For $1 \leq i<j \leq n$, put $X_{i j}:=P_{i} P_{i+1} \cdots P_{j-1}$. Then $X_{i j}$ 's form a basis of $\operatorname{Ker}\left(\phi_{n}: \mathcal{B}_{n}(k) \rightarrow k^{n}\right)$. Using the surjectivity of $\left.\phi_{n}\right|_{\mathcal{A}}$ again, we have $\mathcal{A}=\mathcal{B}_{n}(k)$.

Lemma 3.3. Let $k$ be a field. Let $v_{1}, v_{2}, \ldots, v_{m}$ be $m$ elements of the $k$ algebra $k^{n}$. Then $v_{1}, v_{2}, \ldots, v_{m}$ generate $k^{n}$ as a $k$-algebra if and only if for each $1 \leq i \neq j \leq n$ there exists $v_{\ell}$ whose $i$-th entry and $j$-th entry are distinct.

Proof. Let $A$ be the subalgebra of $k^{n}$ generated by $v_{1}, v_{2}, \ldots, v_{m}$. First we show the "if" part. From the assumption, for each $1 \leq i \leq n$ and for $j \neq i$ we have $w_{i j} \in A$ whose $i$-th entry and $j$-th entry are 1 and 0 , respectively. Since $\prod_{j \neq i} w_{i j}$ is $e_{i}=(0, \ldots, 1, \ldots, 0)$, we see that $A=k^{n}$.

Next we show that the "only if" part. Suppose that $A=k^{n}$ and that there exist $1 \leq i \neq j \leq n$ such that the $i$-th entry and the $j$-th entry of any $v_{\ell}$ coincide. Then $A$ is contained in $\left\{\left(a_{1}, \ldots, a_{n}\right) \in k^{n} \mid a_{i}=a_{j}\right\}$, which is a contradiction.

Let $A_{1}, \ldots, A_{m}$ be $m$ upper triangular matrices of $\mathrm{M}_{n}(k)$. Put

$$
A_{i}=\left(\begin{array}{ccccc}
a(i)_{11} & a(i)_{12} & a(i)_{13} & \cdots & a(i)_{1 n} \\
0 & a(i)_{22} & a(i)_{23} & \cdots & a(i)_{2 n} \\
0 & 0 & a(i)_{33} & \ddots & \vdots \\
\vdots & \vdots & \ddots & \ddots & \vdots \\
0 & 0 & 0 & 0 & a(i)_{n n}
\end{array}\right) .
$$

We define the vectors $w_{i, j}$ for $1 \leq i \leq j \leq n$ by

$$
w_{i, j}=\left(a(1)_{i, j}, a(2)_{i, j}, \ldots, a(m)_{i, j}\right) .
$$

Under this situation, we have the following proposition:

Proposition 3.4. For $m$ upper triangular matrices $A_{1}, \ldots, A_{m}$ of $\mathrm{M}_{n}(k)$, they generate $\mathcal{B}_{n}(k)$ if and only if $w_{11}, w_{22}, \ldots, w_{n n}$ are distinct vectors, and two vectors $w_{i i}-w_{i+1, i+1}$ and $w_{i, i+1}$ are linearly independent for $1 \leq i \leq$ $n-1$.

Proof. By Lemma 3.2 we see that $m$ upper triangular matrices $A_{1}, \ldots, A_{m}$ generate $\mathcal{B}_{n}(k)$ if and only if $\phi_{n}\left(A_{1}\right), \ldots, \phi_{n}\left(A_{m}\right)$ generate $k^{n}$ and $p_{i, i+1}\left(A_{1}\right)$, 
$\ldots, p_{i, i+1}\left(A_{m}\right)$ generate $\mathcal{B}_{2}(k)$ for each $1 \leq i \leq n-1$. From Lemma 3.3, $\phi_{n}\left(A_{1}\right), \ldots, \phi_{n}\left(A_{m}\right)$ generate $k^{n}$ if and only if $w_{11}, w_{22}, \ldots, w_{n n}$ are distinct vectors. By using Lemma 3.1 we easily check that $p_{i, i+1}\left(A_{1}\right), \ldots, p_{i, i+1}\left(A_{m}\right)$ generate $\mathcal{B}_{2}(k)$ if and only if $w_{i i}-w_{i+1, i+1}$ and $w_{i, i+1}$ are linearly independent. Hence we can prove the statement.

3.2. Description of $\mathbf{B}_{\boldsymbol{n}}(\boldsymbol{m})_{\boldsymbol{B}}$. In this subsection, we describe $\mathrm{B}_{n}(m)_{B}$ explicitly by using the configuration space of the affine space. Note that $\mathrm{B}_{n}(m)_{B}$ is the scheme of $m$ upper triangular $n \times n$ matrices which generate the algebra of upper triangular matrices.

Definition 3.5. We define the configuration space $F_{n}(X)$ of a scheme $X$ by

$$
F_{n}(X):=\left\{\left(p_{1}, p_{2}, \ldots, p_{n}\right) \in X^{n} \mid p_{i} \neq p_{j} \text { for } i \neq j\right\} .
$$

For example, we denote by $F_{n}\left(\mathbb{A}_{\mathbb{Z}}^{m}\right)$ the configuration space of ordered distinct $n$-points in $\mathbb{A}_{\mathbb{Z}}^{m}$.

Let $A_{1}, A_{2}, \ldots, A_{m}, w_{i, j}$ be as in (1) and (2). We define the morphism $\Phi_{n, m}: \mathrm{B}_{n}(m)_{B} \rightarrow F_{n}\left(\mathbb{A}_{\mathbb{Z}}^{m}\right)$ by $\left(A_{1}, \ldots, A_{m}\right) \mapsto\left(w_{11}, w_{22}, \ldots, w_{n n}\right)$. The morphism $\Phi_{n, m}$ is well-defined by Proposition 3.4. Let us denote $\mathrm{B}_{n}\left(\Upsilon_{m}\right)$ by $\mathrm{B}_{n}(m)$. We define the isomorphism $\Xi_{n, m}: \mathrm{B}_{n}(m) \rightarrow\left(\mathbb{A}_{\mathbb{Z}}^{m}\right)^{n} \times\left(\mathbb{A}_{\mathbb{Z}}^{m}\right)^{n-1} \times$ $\left(\mathbb{A}_{\mathbb{Z}}^{m}\right)^{(n-1)(n-2) / 2}$ by

$$
\left(A_{1}, \ldots, A_{m}\right) \mapsto\left(\left(w_{11}, w_{22}, \ldots, w_{n n}\right),\left(w_{12}, w_{23}, \ldots, w_{n-1, n}\right),\left(w_{i, j}\right)_{|i-j| \geq 2}\right) .
$$

Under these preparations, we obtain:

Proposition 3.6. Let $n$ be an integer with $n \geq 2$. The morphism $\Phi_{n, m}$ : $\mathrm{B}_{n}(m)_{B} \rightarrow F_{n}\left(\mathbb{A}_{\mathbb{Z}}^{m}\right)$ is a fibre bundle with fibre $\left(\mathbb{A}_{\mathbb{Z}}^{m} \backslash \mathbb{A}_{\mathbb{Z}}^{1}\right)^{n-1} \times \mathbb{A}_{\mathbb{Z}}^{m(n-2)(n-1) / 2}$. More precisely, there exists a Zariski open covering $F_{n}\left(\mathbb{A}_{\mathbb{Z}}^{m}\right)=\cup_{i \in I} U_{i}$ such that $\Phi_{n, m}^{-1}\left(U_{i}\right) \cong U_{i} \times\left(\mathbb{A}_{\mathbb{Z}}^{m} \backslash \mathbb{A}_{\mathbb{Z}}^{1}\right)^{n-1} \times\left(\mathbb{A}_{\mathbb{Z}}^{m}\right)^{(n-2)(n-1) / 2}$ and the structure group is $G:=\underbrace{G_{0} \times \cdots \times G_{0}}_{n-1}$, where

$$
G_{0}:=\left\{\left(\begin{array}{cccc}
1 & * & \cdots & * \\
0 & * & \cdots & * \\
\vdots & \vdots & \ddots & \vdots \\
0 & * & \cdots & *
\end{array}\right) \in \mathrm{GL}_{m}\right\}
$$

Proof. Set $\mathbb{A}_{\mathbb{Z}}^{m} \backslash \mathbb{A}_{\mathbb{Z}}^{1}:=\left\{\left(t_{1}, t_{2}, \ldots, t_{m}\right) \in \mathbb{A}_{\mathbb{Z}}^{m} \mid\left(t_{2}, \ldots, t_{m}\right) \neq(0, \ldots, 0)\right\}$. Let $G_{0}$ act on $\mathbb{A}_{\mathbb{Z}}^{m} \backslash \mathbb{A}_{\mathbb{Z}}^{1}$ by ${ }^{t}\left(t_{1}, t_{2}, \ldots, t_{m}\right) \mapsto A^{t}\left(t_{1}, t_{2}, \ldots, t_{m}\right)$. Then we define the action of $G$ on $\left(\mathbb{A}_{\mathbb{Z}}^{m} \backslash \mathbb{A}_{\mathbb{Z}}^{1}\right)^{n-1} \times\left(\mathbb{A}_{\mathbb{Z}}^{m}\right)^{(n-2)(n-1) / 2}$ by

$$
\left(\left(z_{1}, \ldots, z_{n-1}\right),\left(w_{i j}\right)_{|i-j| \geq 2}\right) \mapsto\left(\left(A_{1} z_{1}, \ldots, A_{n-1} z_{n-1}\right),\left(w_{i j}\right)_{|i-j| \geq 2}\right)
$$

for $\left(A_{1}, \ldots, A_{n-1}\right) \in G=G_{0} \times \cdots \times G_{0}$. 
For each point of $F_{n}\left(\mathbb{A}_{\mathbb{Z}}^{m}\right)$, we have a neighbourhood $U$ such that there exist $n-1$ bases

$$
\begin{gathered}
\left\{v_{1}-v_{2}, u(1)_{2}, \ldots, u(1)_{m}\right\}, \\
\left\{v_{2}-v_{3}, u(2)_{2}, \ldots, u(2)_{m}\right\}, \\
\ldots \\
\left\{v_{n-1}-v_{n}, u(n-1)_{2}, \ldots, u(n-1)_{m}\right\}
\end{gathered}
$$

of $U$-valued points of $\mathbb{A}_{\mathbb{Z}}^{m}$ for $\left(v_{1}, \ldots, v_{n}\right) \in U$. We define the isomorphism $U \times\left(\mathbb{A}_{\mathbb{Z}}^{m} \backslash \mathbb{A}_{\mathbb{Z}}^{1}\right)^{n-1} \times\left(\mathbb{A}_{\mathbb{Z}}^{m}\right)^{(n-2)(n-1) / 2} \rightarrow \Phi_{n, m}^{-1}(U)$ by

$$
\begin{aligned}
&\left(\left(v_{1}, \ldots, v_{n}\right),\left(t(i)_{1}, \ldots, t(i)_{m}\right)_{1 \leq i \leq n-1},\left(w_{i j}\right)_{|i-j| \geq 2}\right) \\
& \mapsto \Xi_{n, m}^{-1}\left(\left(v_{1}, \ldots, v_{n}\right),\left(w_{12}, \ldots, w_{n-1, n}\right),\left(w_{i j}\right)_{|i-j| \geq 2}\right),
\end{aligned}
$$

where $w_{i, i+1}:=t(i)_{1}\left(v_{i}-v_{i+1}\right)+t(i)_{2} u(i)_{2}+\cdots+t(i)_{m} u(i)_{m}$ for $1 \leq i \leq n-1$. Thus we easily see that $\Phi_{n, m}$ is a fiber bundle with the structure group $G$.

Remark 3.7. We remark that if $m=1$, then $\mathrm{B}_{n}(1)_{B}$ is empty. Hence $\mathrm{Ch}_{n}(1)_{B}$ and $\operatorname{Rep}_{n}(1)_{B}$ are also empty. If $n=1$, then $\operatorname{Rep}_{1}(m)=\mathrm{B}_{1}(m)_{B}=$ $\mathrm{Ch}_{1}(m)_{B}=\mathbb{A}_{\mathbb{Z}}^{m}$. Therefore in the sequel we assume that $n, m \geq 2$.

3.3. Description of $\mathbf{C h}_{\boldsymbol{n}}(\boldsymbol{m})_{B}$. In this subsection, we describe the moduli of representations with Borel mold $\mathrm{Ch}_{n}(m)_{B}$ explicitly.

The morphism $\Phi_{n, m}: \mathrm{B}_{n}(m)_{B} \rightarrow F_{n}\left(\mathbb{A}_{\mathbb{Z}}^{m}\right)$ is $\mathrm{B}_{n}$-equivariant. Here the group scheme $\mathrm{B}_{n}$ acts on $F_{n}\left(\mathbb{A}_{\mathbb{Z}}^{m}\right)$ trivially. Hence $\Phi_{n, m}$ induces $\Psi_{n, m}$ : $\mathrm{Ch}_{n}(m)_{B} \rightarrow F_{n}\left(\mathbb{A}_{\mathbb{Z}}^{m}\right)$. For each point of $F_{n}\left(\mathbb{A}_{\mathbb{Z}}^{m}\right)$, we take an open neighbourhood $U$ as in the proof of Proposition 3.6.

Let us consider the action of $\mathrm{B}_{n}$ on $\Phi_{n, m}^{-1}(U) \cong U \times\left(\mathbb{A}_{\mathbb{Z}}^{m} \backslash \mathbb{A}_{\mathbb{Z}}^{1}\right)^{n-1} \times$ $\mathbb{A}_{\mathbb{Z}}^{m(n-2)(n-1) / 2}$. Let $x=\left(\left(v_{1}, \ldots, v_{n}\right),\left(t(i)_{1}, \ldots, t(i)_{m}\right)_{1 \leq i \leq n-1},\left(w_{i j}\right)_{|i-j| \geq 2}\right)$ $\in U \times\left(\mathbb{A}_{\mathbb{Z}}^{m} \backslash \mathbb{A}_{\mathbb{Z}}^{1}\right)^{n-1} \times \mathbb{A}_{\mathbb{Z}}^{m(n-2)(n-1) / 2}$. For $B=\left(b_{i j}\right) \in \mathrm{B}_{n}$, set $B^{-1}=\left(b_{i j}^{\prime}\right)$. We denote $B \cdot x$ by $\left(\left(v_{1}^{\prime}, \ldots, v_{n}^{\prime}\right),\left(t^{\prime}(i)_{1}, \ldots, t^{\prime}(i)_{m}\right)_{1 \leq i \leq n-1},\left(w_{i j}^{\prime}\right)_{|i-j| \geq 2}\right)$. Then we have

$$
\begin{aligned}
v_{i}^{\prime} & =v_{i} \\
t^{\prime}(i)_{1} & =-\frac{b_{i, i+1}}{b_{i+1, i+1}}+\frac{b_{i i}}{b_{i+1, i+1}} t(i)_{1}, \\
t^{\prime}(i)_{2} & =\frac{b_{i i}}{b_{i+1, i+1}} t(i)_{2}, \\
& \cdots \\
t^{\prime}(i)_{m} & =\frac{b_{i i}}{b_{i+1, i+1}} t(i)_{m}, \\
w_{i j}^{\prime} & =\sum_{i \leq k \leq \ell \leq j} b_{i k} w_{k \ell} b_{\ell j}^{\prime} .
\end{aligned}
$$


By calculating $w_{i j}^{\prime}$, we have

$$
\begin{aligned}
w_{i j}^{\prime}= & b_{i i} w_{i j} b_{j j}^{\prime}+\sum_{i \leq k \leq j} b_{i k} w_{k k} b_{k j}^{\prime}+(\text { the other terms }) \\
= & b_{i i} w_{i j} b_{j j}^{\prime}-b_{i j} b_{j j}^{\prime}\left(w_{j-1, j-1}-w_{j j}\right) \\
& -\left(b_{i, j-1} b_{j-1, j}^{\prime}+b_{i j} b_{j j}^{\prime}\right)\left(w_{j-2, j-2}-w_{j-1, j-1}\right) \\
& -\left(b_{i, j-2} b_{j-2, j}^{\prime}+b_{i, j-1} b_{j-1, j}^{\prime}+b_{i j} b_{j j}^{\prime}\right)\left(w_{j-3, j-3}-w_{j-2, j-2}\right)-\cdots \\
& -\left(b_{i, i+1} b_{i+1, j}^{\prime}+\cdots+b_{i j} b_{j j}^{\prime}\right)\left(w_{i i}-w_{i+1, i+1}\right)+\left(\sum_{i \leq k \leq j} b_{i k} b_{k j}^{\prime}\right) w_{i i} \\
& +(\text { the other terms }) \\
= & b_{i i} w_{i j} b_{j j}^{\prime}-b_{i j} b_{j j}^{\prime}\left(v_{j-1}-v_{j}\right)-\left(b_{i, j-1} b_{j-1, j}^{\prime}+b_{i j} b_{j j}^{\prime}\right)\left(v_{j-2}-v_{j-1}\right) \\
& -\left(b_{i, j-2} b_{j-2, j}^{\prime}+b_{i, j-1} b_{j-1, j}^{\prime}+b_{i j} b_{j j}^{\prime}\right)\left(v_{j-3}-v_{j-2}\right)-\cdots \\
& -\left(b_{i, i+1} b_{i+1, j}^{\prime}+\cdots+b_{i j} b_{j j}^{\prime}\right)\left(v_{i}-v_{i+1}\right)+(\text { the other terms }) .
\end{aligned}
$$

Here we used the equality $\sum_{i \leq k \leq j} b_{i k} b_{k j}^{\prime}=\delta_{i j}=0$ and we denoted $v_{k}$ by $w_{k k}$.

We define a morphism $\Phi_{n, m}^{-1}(U) \cong U \times\left(\mathbb{A}_{\mathbb{Z}}^{m} \backslash \mathbb{A}_{\mathbb{Z}}^{1}\right)^{n-1} \times \mathbb{A}_{\mathbb{Z}}^{m(n-2)(n-1) / 2} \rightarrow$ $U \times\left(\mathbb{P}_{\mathbb{Z}}^{m-2}\right)^{n-1} \times\left(\mathbb{A}_{\mathbb{Z}}^{m-1}\right)^{(n-2)(n-1) / 2}$ by

$$
\begin{aligned}
& \left(\left(v_{1}, \ldots, v_{n}\right),\left(t(i)_{1}, \ldots, t(i)_{m}\right)_{1 \leq i \leq n-1},\left(w_{i j}\right)_{|i-j| \geq 2}\right) \\
& \quad \mapsto\left(\left(v_{1}, \ldots, v_{n}\right),\left(t(i)_{2}: \cdots: t(i)_{m}\right)_{1 \leq i \leq n-1},\left(\bar{w}_{i j}\right)_{|i-j| \geq 2}\right),
\end{aligned}
$$

where $\bar{w}_{i j} \in \mathbb{A}_{\mathbb{Z}}^{m-1}=\left\langle u_{i j}(2), \ldots, u_{i j}(m)\right\rangle \subset \mathbb{A}_{\mathbb{Z}}^{m}=\left\langle\left(v_{j-1}-v_{j}\right), u_{i j}(2), \ldots\right.$, $\left.u_{i j}(m)\right\rangle$ is defined as follows: We take $\left(v_{j-1}-v_{j}\right), u_{i j}(2), \ldots, u_{i j}(m)$ as a basis of $U \times \mathbb{A}_{\mathbb{Z}}^{m}$ over $U$. The above calculation follows that $w_{i j}^{\prime}=$ $b_{i i} w_{i j} b_{j j}^{\prime}-b_{i j} b_{j j}^{\prime}\left(v_{j-1}-v_{j}\right)+\cdots$, and hence by choosing suitable $b_{i j}$, we can assume that $w_{i j}^{\prime} \in\left\langle u_{i j}(2), \ldots, u_{i j}(m)\right\rangle$. Then we put $\bar{w}_{i j}=w_{i j}^{\prime}$. Let $B_{n}$ act on $U \times\left(\mathbb{P}_{\mathbb{Z}}^{m-2}\right)^{n-1} \times\left(\mathbb{A}_{\mathbb{Z}}^{m-1}\right)^{(n-2)(n-1) / 2}$ trivially. Then the above morphism is $\mathrm{B}_{n}$-equivariant. Since the pull-back $\Psi_{n, m}^{-1}(U) \subseteq \mathrm{Ch}_{n}(m)_{B}$ of $U$ is a universal geometric quotient of $\Phi_{n, m}^{-1}(U)$ by $\mathrm{B}_{n}$, the morphism $\Phi_{n, m}^{-1}(U) \rightarrow U \times\left(\mathbb{P}_{\mathbb{Z}}^{m-2}\right)^{n-1} \times\left(\mathbb{A}_{\mathbb{Z}}^{m-1}\right)^{(n-2)(n-1) / 2}$ induces a morphism $\varphi:$ $\Psi_{n, m}^{-1}(U) \rightarrow U \times\left(\mathbb{P}_{\mathbb{Z}}^{m-2}\right)^{n-1} \times\left(\mathbb{A}_{\mathbb{Z}}^{m-1}\right)^{(n-2)(n-1) / 2}$. We can easily check that $\varphi$ gives a bijection between geometric points. The schemes $\Psi_{n, m}^{-1}(U)$ and $U \times\left(\mathbb{P}_{\mathbb{Z}}^{m-2}\right)^{n-1} \times\left(\mathbb{A}_{\mathbb{Z}}^{m-1}\right)^{(n-2)(n-1) / 2}$ are smooth over $\mathbb{Z}$. Because $\varphi$ is birational, it is an isomorphism by Zariski's Main Theorem. Therefore we have:

Proposition 3.8. The morphism $\Psi_{n, m}: \mathrm{Ch}_{n}(m)_{B} \rightarrow F_{n}\left(\mathbb{A}_{\mathbb{Z}}^{m}\right)$ is a fibre bundle with fibre $\left(\mathbb{P}_{\mathbb{Z}}^{m-2}\right)^{n-1} \times\left(\mathbb{A}_{\mathbb{Z}}^{m-1}\right)^{(n-2)(n-1) / 2}$. 
Remark 3.9. The morphism $\Psi_{n, m}: \mathrm{Ch}_{n}(m)_{B} \rightarrow F_{n}\left(\mathbb{A}_{\mathbb{Z}}^{m}\right)$ can be interpreted as follows: Let $\mathrm{Ch}_{1}(m)$ be the moduli of characters for the free monoid $\Upsilon_{m}$ of rank $m$. The scheme $F_{n}\left(\mathbb{A}_{\mathbb{Z}}^{m}\right)$ is isomorphic to the configuration space $F_{n}\left(\mathrm{Ch}_{1}(m)\right)$ of $\mathrm{Ch}_{1}(m)$ defined by $F_{n}\left(\mathrm{Ch}_{1}(m)\right):=\left\{\left(\chi_{1}, \ldots, \chi_{n}\right) \in\right.$ $\mathrm{Ch}_{1}(m) \mid \chi_{i} \neq \chi_{j}$ for $\left.i \neq j\right\}$, since $\mathrm{Ch}_{1}(m) \cong \mathbb{A}_{\mathbb{Z}}^{m}$. For $\rho \in \mathrm{B}_{n}(m)_{B}$ we can define $\left(\rho_{11}, \rho_{22}, \ldots, \rho_{n n}\right) \in F_{n}\left(\mathrm{Ch}_{1}(m)\right)$. This correspondence induces $\Psi_{n, m}: \mathrm{Ch}_{n}(m)_{B} \rightarrow F_{n}\left(\mathbb{A}_{\mathbb{Z}}^{m}\right) \cong F_{n}\left(\mathrm{Ch}_{1}(m)\right)$. The fibre $\Psi_{n, m}^{-1}\left(\chi_{1}, \ldots, \chi_{n}\right)$ corresponds to the equivalence classes of representations with Borel mold which are extensions of characters $\left(\chi_{1}, \ldots, \chi_{n}\right)$.

In the case $n=2$, Proposition 3.8 says that $\Psi_{2, m}: \mathrm{Ch}_{2}(m)_{B} \rightarrow F_{2}\left(\mathbb{A}_{\mathbb{Z}}^{m}\right)$ is a fibre bundle with fibre $\mathbb{P}_{\mathbb{Z}}^{m-2}$. In particular, $\mathrm{Ch}_{2}(2)_{B}$ is isomorphic to $F_{2}\left(\mathbb{A}_{\mathbb{Z}}^{2}\right) \cong \mathbb{A}_{\mathbb{Z}}^{2} \times\left(\mathbb{A}_{\mathbb{Z}}^{2} \backslash\{0\}\right)$. Let us describe the $\mathbb{P}_{\mathbb{Z}}^{m-2}$-bundle over $F_{2}\left(\mathbb{A}_{\mathbb{Z}}^{m}\right)$ more precisely.

The configuration space $F_{2}\left(\mathbb{A}_{\mathbb{Z}}^{m}\right)$ is isomorphic to $\mathbb{A}_{\mathbb{Z}}^{m} \times\left(\mathbb{A}_{\mathbb{Z}}^{m} \backslash\{0\}\right)$ by $\left(v_{1}, v_{2}\right) \mapsto\left(v_{1}, v_{1}-v_{2}\right)$. We denote by $f$ the composition of morphisms

$$
F_{2}\left(\mathbb{A}_{\mathbb{Z}}^{m}\right) \cong \mathbb{A}_{\mathbb{Z}}^{m} \times\left(\mathbb{A}_{\mathbb{Z}}^{m} \backslash\{0\}\right) \rightarrow \mathbb{A}_{\mathbb{Z}}^{m} \backslash\{0\} \rightarrow \mathbb{P}_{\mathbb{Z}}^{m-1}
$$

Let us consider the short exact sequence

$$
0 \rightarrow \mathcal{O}_{\mathbb{P}_{\mathbb{Z}}^{m-1}}(-1) \rightarrow \mathcal{O}_{\mathbb{P}_{\mathbb{Z}}^{m-1}}^{\oplus m} \rightarrow T_{\mathbb{P}_{\mathbb{Z}}^{m-1}}(-1) \rightarrow 0
$$

on $\mathbb{P}_{\mathbb{Z}}^{m-1}$. Put $\mathcal{E}:=f^{*} T_{\mathbb{P}_{\mathbb{Z}}^{m-1}}(-1)$. The morphism $\Psi_{2, m}: \mathrm{Ch}_{2}(m)_{B} \rightarrow$ $F_{2}\left(\mathbb{A}_{\mathbb{Z}}^{m}\right)$ is described as follows:

Proposition 3.10. The moduli $\mathrm{Ch}_{2}(m)_{B}$ is isomorphic to $\mathcal{P}$ roj $\mathcal{E}^{\vee}$ over $F_{2}\left(\mathbb{A}_{\mathbb{Z}}^{m}\right)$.

Proof. Recall that the morphism $\Psi_{2, m}: \mathrm{Ch}_{2}(m)_{B} \rightarrow F_{2}\left(\mathbb{A}_{\mathbb{Z}}^{m}\right)$ is given by $\left[\left(A_{1}, \ldots, A_{m}\right)\right] \mapsto\left(w_{11}, w_{22}\right)$, where $A_{i}, w_{i j}$ are as in (1) and (2). Let us consider the pull-back of (3) by $f \circ \Psi_{2, m}$ :

$$
0 \rightarrow\left(f \circ \Psi_{2, m}\right)^{*} \mathcal{O}_{\mathbb{P}_{\mathbb{Z}}^{m-1}}(-1) \stackrel{w_{11-w_{22}}}{\longrightarrow} \mathcal{O}_{\mathrm{Ch}_{2}(m)_{B}}^{\oplus m} \rightarrow \Psi_{2, m}^{*} \mathcal{E} \rightarrow 0 .
$$

The vectors $w_{12}$ and $w_{11}-w_{22}$ are linearly independent. For $B=\left(b_{i j}\right) \in \mathrm{B}_{2}$, the $w_{12}$ vector of $B \cdot\left(A_{1}, \ldots, A_{m}\right)$ is given by $-b_{12} / b_{22} \cdot\left(w_{11}-w_{22}\right)+b_{11} / b_{22}$. $w_{12}$. From these facts, the vector $w_{12}$ determines a sub-line bundle $\mathcal{L}$ of $\Psi_{2, m}^{*} \mathcal{E}$. Hence we have the surjection $\Psi_{2, m}^{*} \mathcal{E}^{\vee} \rightarrow \mathcal{L}^{\vee} \rightarrow 0$. The surjective homomorphism of algebras $S\left(\Psi_{2, m}^{*} \mathcal{E}^{\vee}\right) \rightarrow S\left(\mathcal{L}^{\vee}\right)$ induces $\mathrm{Ch}_{2}(m)_{B} \rightarrow$ $\mathrm{Ch}_{2}(m)_{B} \times \mathcal{P}$ roj $\mathcal{E}^{\vee} \stackrel{\text { proj. }}{\longrightarrow} \operatorname{Proj} \mathcal{E}^{\vee}$. We can easily check that this is an isomorphism.

3.4. Description of $\operatorname{Rep}_{\boldsymbol{n}}(\boldsymbol{m})_{\boldsymbol{B}}$. In this subsection, we describe $\operatorname{Rep}_{n}(m)_{B}$. 
In $\S 2$ we obtained a diagram which is a fibre product:

$$
\begin{array}{ccc}
\mathrm{B}_{n}(m)_{B} \times \mathrm{PGL}_{n} & \stackrel{f}{\rightarrow} & \operatorname{Rep}_{n}(m)_{B} \\
\downarrow p_{1} & & \downarrow \pi \\
\mathrm{B}_{n}(m)_{B} & \stackrel{\pi^{\prime}}{\rightarrow} & \mathrm{Ch}_{n}(m)_{B},
\end{array}
$$

where $f: \mathrm{B}_{n}(m)_{B} \times \mathrm{PGL}_{n} \rightarrow \operatorname{Rep}_{n}(m)_{B}$ is given by $(\rho, P) \mapsto P^{-1} \rho P$ and $p_{1}$ is the first projection. The group scheme $\mathrm{B}_{n}$ acts on $\mathrm{B}_{n}(m)_{B} \times \mathrm{PGL}_{n}$ by $(\rho, P) \mapsto\left(Q \rho Q^{-1}, Q P\right)$. The morphism $f$ is a $\mathrm{B}_{n}$-principal fibre bundle. Hence we conclude that $\mathrm{B}_{n}(m)_{B} \times_{\mathrm{B}_{n}} \mathrm{PGL}_{n} \cong \operatorname{Rep}_{n}(m)_{B}$.

The universal representation with Borel mold on $\operatorname{Rep}_{n}(m)_{B}$ induces the action of the free monoid $\Upsilon_{m}$ on the trivial bundle $\mathcal{O}_{\operatorname{Rep}_{n}(m)_{B}}^{\oplus n}$. In [Na2] we obtained a unique complete flag $0 \subset \mathcal{E}_{1} \subset \cdots \subset \mathcal{E}_{n-1} \subset \mathcal{O}_{\operatorname{Rep}_{n}(m)_{B}}^{\oplus n}$ such that $\mathcal{E}_{i}$ is a unique $\Upsilon_{m}$-invariant subbundle of rank $i$. Then we get a morphism $\operatorname{Rep}_{n}(m)_{B} \rightarrow \operatorname{Flag}\left(\mathbb{A}_{\mathbb{Z}}^{n}\right)$ associated to the complete flag, where $\operatorname{Flag}\left(\mathbb{A}_{\mathbb{Z}}^{n}\right)$ is the flag scheme consisting of complete flags of the rank $n$ trivial bundle.

Proposition 3.11. The morphism $\operatorname{Rep}_{n}(m)_{B} \rightarrow \operatorname{Flag}\left(\mathbb{A}_{\mathbb{Z}}^{n}\right)$ is a fibre bundle with fibre $\mathrm{B}_{n}(m)_{B}$.

Proof. For each $x \in \operatorname{Flag}\left(\mathbb{A}_{\mathbb{Z}}^{n}\right)$, we can choose an open neighbourhood $U$ of $x$ and $n$ sections $s_{i}(1 \leq i \leq n)$ of $\mathcal{O}_{U}^{\oplus n}$ such that $\oplus_{i=1}^{k} \mathcal{O}_{U} \cdot s_{i}$ is the rank $k$ subbundle of the universal flag on $U$. We denote by $\widetilde{U}$ the inverse image of $U$ by $\operatorname{Rep}_{n}(m)_{B} \rightarrow \operatorname{Flag}\left(\mathbb{A}_{\mathbb{Z}}^{n}\right)$. Let $\mathcal{E}_{*}$ be the pull-back of the universal flag on $\widetilde{U}$. Let $\widetilde{s}_{i}$ be the pull-back of $s_{i}$. Then we define a morphism $U \times \mathrm{B}_{n}(m)_{B} \rightarrow$ $\widetilde{U}$ by corresponding $\left(\mathcal{E}_{*}, \rho\right)$ to the representation $\rho$ with respect to the basis $\left\{\widetilde{s}_{i}\right\}$ (not the canonical basis!). We can easily check that $U \times \mathrm{B}_{n}(m)_{B} \rightarrow \widetilde{U}$ is an isomorphism, which completes the proof. The statement can be also verified by the fact that $\mathrm{B}_{n}(m)_{B} \times_{\mathrm{B}_{n}} \mathrm{PGL}_{n} \cong \operatorname{Rep}_{n}(m)_{B}$.

\section{Cohomology of $\mathrm{B}_{n}(m)_{B}$.}

In $\S 3.2$ we described the scheme $\mathrm{B}_{n}(m)_{B}$ over $\mathbb{Z}$ as a fibre bundle over the configuration space $F_{n}\left(\mathbb{A}_{\mathbb{Z}}^{m}\right)$. In the rest of this paper we abbreviate the $\mathbb{C}$ valued point of $\mathrm{B}_{n}(m)_{B}$ with classical topology to $\mathrm{B}_{n}(m)_{B}$. In this section we calculate the cohomology ring of $\mathrm{B}_{n}(m)_{B}$ for $m \geq 2$ by using the Serre spectral sequence associated with the fibre bundle. For a topological space $X$, we denote by $H^{q}(X)$ the integral cohomology group $H^{q}(X ; \mathbb{Z})$.

First, we recall the cohomology ring of the configuration space $F_{n}\left(\mathbb{R}^{m}\right)$ (cf. [Co1] and [Co2]). Let $F_{n}\left(\mathbb{R}^{m}\right)$ be the configuration space of ordered distinct $n$-points in $\mathbb{R}^{m}$ :

$$
F_{n}\left(\mathbb{R}^{m}\right)=\left\{\left(x_{1}, \ldots, x_{n}\right) \in\left(\mathbb{R}^{m}\right)^{n} \mid x_{i} \neq x_{j}(i \neq j)\right\} .
$$

Since $F_{2}\left(\mathbb{R}^{m}\right)$ is homotopy equivalent to the $(m-1)$-sphere $S^{m-1}$, we have $H^{*}\left(F_{2}\left(\mathbb{R}^{m}\right)\right) \cong \Lambda(s)$ where the degree $|s|=m-1$. For $i \neq j$, we define 
the map $\pi_{i, j}: F_{n}\left(\mathbb{R}^{m}\right) \rightarrow F_{2}\left(\mathbb{R}^{m}\right)$ given by $\pi_{i, j}\left(x_{1}, \ldots, x_{n}\right)=\left(x_{i}, x_{j}\right)$. Let $s(i, j)=\pi_{i, j}^{*}(s)$. Then we have $s(i, j)^{2}=0$ and $s(j, i)=(-1)^{m} s(i, j)$.

Theorem 4.1 (cf. [Co1] and [Co2]). The cohomology ring of the configuration space $F_{n}\left(\mathbb{R}^{m}\right)$ is a graded commutative associative ring generated by $s(i, j)$ for $1 \leq i<j \leq n$ with a complete set of relations:

$$
\begin{aligned}
s(i, j)^{2} & =0, \\
s(i, k) s(j, k) & =s(i, j) s(j, k)-s(i, j) s(i, k) \text { for } i<j<k .
\end{aligned}
$$

By Proposition 3.6, there is a fibre bundle

$$
Y_{B} \stackrel{i}{\longrightarrow} \mathrm{B}_{n}(m)_{B} \stackrel{\Phi_{n, m}}{\longrightarrow} F_{n}\left(\mathbb{C}^{m}\right),
$$

where the fibre $Y_{B}$ is $\left(\mathbb{C}^{m}-\mathbb{C}^{1}\right)^{n-1} \times \mathbb{C}^{m(n-1)(n-2) / 2}$. Since $Y_{B}$ is homotopy equivalent to the product of spheres:

$$
Y_{B} \simeq \overbrace{S^{2 m-3} \times \cdots \times S^{2 m-3}}^{n-1},
$$

the cohomology of the fibre $Y_{B}$ is given by

$$
H^{*}\left(Y_{B}\right) \cong \Lambda\left(s_{1}^{\prime}, \ldots, s_{n-1}^{\prime}\right),
$$

where the degree of $s_{j}^{\prime}$ is $2 m-3$ for $j=1, \ldots, n-1$.

Lemma 4.2. $\mathrm{B}_{n}(m)_{B}$ is $(2 m-4)$-connected.

Proof. Note that the configuration space $F_{n}\left(\mathbb{C}^{m}\right)$ is $(2 m-2)$-connected. Then the lemma follows from the long exact sequence of homotopy groups associated with the fibre bundle (4).

There is a Serre spectral sequence associated with the fibre bundle (4)

$$
E_{2}^{p, q}=H^{p}\left(F_{n}\left(\mathbb{C}^{m}\right) ; \mathcal{H}^{q}\left(Y_{B}\right)\right) \Longrightarrow H^{p+q}\left(\mathrm{~B}_{n}(m)_{B}\right) .
$$

Note that the coefficient system is trivial, since $F_{n}\left(\mathbb{C}^{m}\right)$ is $(2 m-2)$-connected $(m \geq 2)$. Since $H^{*}\left(F_{n}\left(\mathbb{C}^{m}\right)\right)$ and $H^{*}\left(Y_{B}\right)$ are free over $\mathbb{Z}$, we have an isomorphism

$$
E_{2}^{p, q} \cong H^{p}\left(F_{n}\left(\mathbb{C}^{m}\right)\right) \otimes H^{q}\left(Y_{B}\right) .
$$

By Theorem $4.1, H^{p}\left(F_{n}\left(\mathbb{C}^{m}\right)\right)=0$ for $1 \leq p \leq 2 m-2$. Hence this spectral sequence collapses from $E_{2}$-term. In particular, $H^{*}\left(\mathrm{~B}_{n}(m)_{B}\right)$ is free over $\mathbb{Z}$. Since $i^{*}: H^{2 m-3}\left(\mathrm{~B}_{n}(m)_{B}\right) \rightarrow H^{2 m-3}\left(Y_{B}\right)$ is an isomorphism, there is $s_{j} \in H^{2 m-3}\left(\mathrm{~B}_{n}(m)_{B}\right)$ such that $i^{*}\left(s_{j}\right)=s_{j}^{\prime}$ for $j=1, \ldots, n-1$. By using the ring homomorphism $\Phi_{n, m}^{*}: H^{*}\left(F_{n}\left(\mathbb{C}^{m}\right)\right) \rightarrow H^{*}\left(\mathrm{~B}_{n}(m)_{B}\right)$, we regard $H^{*}\left(\mathrm{~B}_{n}(m)_{B}\right)$ as an algebra over $H^{*}\left(F_{n}\left(\mathbb{C}^{m}\right)\right)$.

Theorem 4.3. The cohomology ring of $\mathrm{B}_{n}(m)_{B}$ is an exterior algebra generated by $s_{1}, \ldots, s_{n-1}$ over $H^{*}\left(F_{n}\left(\mathbb{C}^{m}\right)\right)$ :

$$
H^{*}\left(\mathrm{~B}_{n}(m)_{B}\right) \cong H^{*}\left(F_{n}\left(\mathbb{C}^{m}\right)\right) \otimes \Lambda\left(s_{1}, \ldots, s_{n-1}\right) .
$$


Proof. Since $H^{*}\left(\mathrm{~B}_{n}(m)_{B}\right)$ is free over $\mathbb{Z}, s_{j}^{2}=0$ for $j=1, \ldots, n-1$. There is a ring homomorphism $\phi: \Lambda\left(s_{1}, \ldots, s_{n-1}\right) \rightarrow H^{*}\left(\mathrm{~B}_{n}(m)_{B}\right)$. Then $\phi$ is injective since $i^{*} \circ \phi$ is an isomorphism. We consider the following ring homomorphism:

$$
\Phi_{n, m}^{*} \otimes \phi: H^{*}\left(F_{n}\left(\mathbb{C}^{m}\right)\right) \otimes \Lambda\left(s_{1}, \ldots, s_{j}\right) \longrightarrow H^{*}\left(\mathrm{~B}_{n}(m)_{B}\right) .
$$

Then it is easy to see that $\Phi_{n, m}^{*} \otimes \phi$ is an isomorphism.

For $\left(A_{1}, \ldots, A_{m}\right) \in \mathrm{B}_{n}(m)_{B}$, we recall that $a(i)_{k, l}$ is the $(k, l)$-entry of the $i$ th matrix $A_{i}$. Then they define a vector $w_{k, l}$ in $\mathbb{C}^{m}$ by $w_{k, l}=$ $\left(a(1)_{k, l}, \ldots, a(m)_{k, l}\right)$. We set $\bar{w}_{k}=w_{k, k}-w_{k+1, k+1}$ for $k=1, \ldots, n-1$. Let $\mathrm{B}_{n}(m)_{B}^{\prime}$ be the subspace of $\mathrm{B}_{n}(m)_{B}$ defined as follows:

$$
\begin{aligned}
& \mathrm{B}_{n}(m)_{B}^{\prime} \\
& =\left\{\begin{array}{l|l}
\left(A_{1}, \ldots, A_{m}\right) \in \mathrm{B}_{n}(m)_{B} & \begin{array}{l}
a(i)_{k, l}=0 \\
\left(\bar{w}_{k}, w_{k, k+1}\right)=0 \\
\left\|w_{k, k+1}\right\|=1
\end{array} \quad(1 \leq k \leq n-1), \\
\mid \begin{array}{l}
\mid 1 \leq k \leq n-1)
\end{array}
\end{array}\right\},
\end{aligned}
$$

where $(-,-)$ is the standard Hermitian inner product and $\|-\|$ is the associated norm. Let $T^{n}$ be the $n$-dimensional torus $\overbrace{S^{1} \times \cdots \times S^{1}}^{n}$. Then there is a homomorphism from $T^{n}$ into the diagonal matrices of $\mathrm{B}_{n}(\mathbb{C})$. We denote by $T_{\mathbb{R}}$ the image of this homomorphism. Then $\mathrm{B}_{n}(m)_{B}^{\prime}$ is a $T_{\mathbb{R}^{-}}$ equivariant subspace of $\mathrm{B}_{n}(m)_{B}$ where the action of $T_{\mathbb{R}}$ on $\mathrm{B}_{n}(m)_{B}$ is a restriction of the action of $\mathrm{B}_{n}(\mathbb{C})$. We note that $T_{\mathbb{R}}$ acts on $\mathrm{B}_{n}(m)_{B}^{\prime}$ freely. Then the following lemma is easy:

Lemma 4.4. $\mathrm{B}_{n}(m)_{B}^{\prime} \hookrightarrow \mathrm{B}_{n}(m)_{B}$ is a $T_{\mathbb{R}}$-equivariant homotopy equivalence.

The map from $\mathrm{B}_{n}(m)_{B}^{\prime}$ to $F_{n}\left(\mathbb{C}^{m}\right)$ gives a fibre bundle

$$
Y_{B}^{\prime} \longrightarrow \mathrm{B}_{n}(m)_{B}^{\prime} \longrightarrow F_{n}\left(\mathbb{C}^{m}\right),
$$

where the fibre $Y_{B}^{\prime}$ is the product of spheres:

$$
Y_{B}^{\prime}=\overbrace{S^{2 m-3} \times \cdots \times S^{2 m-3}}^{n-1} .
$$

There is a map of fibre bundles from $Y_{B}^{\prime} \rightarrow \mathrm{B}_{n}(m)_{B}^{\prime} \rightarrow F_{n}\left(\mathbb{C}^{m}\right)$ to $Y_{B} \rightarrow$ $\mathrm{B}_{n}(m)_{B} \rightarrow F_{n}\left(\mathbb{C}^{m}\right)$ which induces homotopy equivalences:

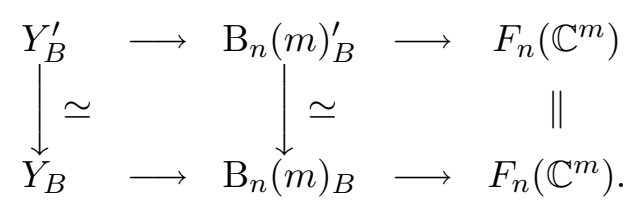




\section{Cohomology of $\mathrm{Ch}_{n}(m)_{B}$.}

In the rest of this paper we abbreviate the $\mathbb{C}$-valued point of $\mathrm{Ch}_{n}(m)_{B}$ with classical topology to $\mathrm{Ch}_{n}(m)_{B}$. In $\S 3.3$ we obtained a description of the scheme $\mathrm{Ch}_{n}(m)_{B}$ over $\mathbb{Z}$ as a fibre bundle over the configuration space $F_{n}\left(\mathbb{A}_{\mathbb{Z}}^{m}\right)$. By using the Serre spectral sequence of the fibre bundle, we calculate the cohomology ring of $\mathrm{Ch}_{n}(m)_{B}$ for $m \geq 2$.

The space $\mathrm{Ch}_{n}(m)_{B}$ is defined to be the quotient space of $\mathrm{B}_{n}(m)_{B}$ by the free action of $\mathrm{B}_{n}(\mathbb{C})$. The torus $T_{\mathbb{R}} \subset \mathrm{B}_{n}(\mathbb{C})$ also acts on $\mathrm{B}_{n}(m)_{B}$. There is a fibre bundle

$$
\mathrm{B}_{n}(\mathbb{C}) / T_{\mathbb{R}} \longrightarrow \mathrm{B}_{n}(m)_{B} / T_{\mathbb{R}} \longrightarrow \mathrm{Ch}_{n}(m)_{B}
$$

Since the fibre $\mathrm{B}_{n}(\mathbb{C}) / T_{\mathbb{R}}$ is contractible, the projection $\mathrm{B}_{n}(m)_{B} / T_{\mathbb{R}} \rightarrow$ $\mathrm{Ch}_{n}(m)_{B}$ is a weak homotopy equivalence. By Lemma 4.4 , there is a $T_{\mathbb{R}^{-}}$ subspace $\mathrm{B}_{n}(m)_{B}^{\prime}$ of $\mathrm{B}_{n}(m)_{B}$ such that the inclusion is a $T_{\mathbb{R}}$-equivariant homotopy equivalence. Let $\mathrm{Ch}_{n}(m)_{B}^{\prime}$ be the quotient space $\mathrm{B}_{n}(m)_{B}^{\prime} / T_{\mathbb{R}}$. Hence we have the following lemma:

Lemma 5.1. $\mathrm{Ch}_{n}(m)_{B}$ is weakly homotopy equivalent to $\mathrm{Ch}_{n}(m)_{B}^{\prime}$.

By Lemma 5.1, the natural map $\mathrm{Ch}_{n}(m)_{B}^{\prime} \rightarrow \mathrm{Ch}_{n}(m)_{B}$ induces an isomorphism of cohomology rings. Hence we calculate the cohomology of $\mathrm{Ch}_{n}(m)_{B}^{\prime}$. There is a map from $\mathrm{Ch}_{n}(m)_{B}^{\prime}$ to $F_{n}\left(\mathbb{C}^{m}\right)$ which gives a fibre bundle

$$
Y_{C}^{\prime} \stackrel{i^{\prime}}{\longrightarrow} \mathrm{Ch}_{n}(m)_{B}^{\prime} \stackrel{\Psi_{n, m}^{\prime}}{\longrightarrow} F_{n}\left(\mathbb{C}^{m}\right)
$$

Note that we have a commutative diagram of fibre bundles

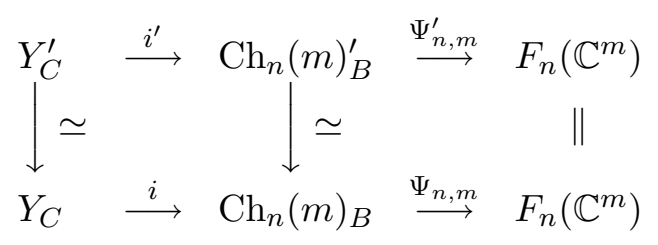

such that the vertical arrows are weak homotopy equivalences. The fibre $Y_{C}^{\prime}$ is the product of complex projective spaces:

$$
Y_{C}^{\prime}=\overbrace{\mathbb{C P}^{m-2} \times \cdots \times \mathbb{C P}^{m-2}}^{n-1} .
$$

Hence we have

$$
H^{*}\left(Y_{C}^{\prime}\right) \cong \mathbb{Z}\left[t_{1}^{\prime}, \ldots, t_{n-1}^{\prime}\right] /\left(t_{1}^{\prime m-1}, \ldots, t_{n-1}^{\prime}{ }^{m-1}\right),
$$

where the degree of $t_{j}^{\prime}$ is 2 for $j=1, \ldots, n-1$. There is a Serre spectral sequence associated with the fibre bundle (5)

$$
E_{2}^{p, q}=H^{p}\left(F_{n}\left(\mathbb{C}^{m}\right) ; \mathcal{H}^{q}\left(Y_{C}^{\prime}\right)\right) \Longrightarrow H^{p+q}\left(\mathrm{Ch}_{n}(m)_{B}^{\prime}\right)
$$


The coefficient system is trivial by the same reason as in the case of $\mathrm{B}_{n}(m)_{B}$. Note that there is an isomorphism

$$
E_{2}^{p, q} \cong H^{p}\left(F_{n}\left(\mathbb{C}^{m}\right)\right) \otimes H^{q}\left(Y_{C}^{\prime}\right),
$$

since $H^{*}\left(F_{n}\left(\mathbb{C}^{m}\right)\right)$ and $H^{*}\left(Y_{C}^{\prime}\right)$ are free over $\mathbb{Z}$. By Theorem 4.1, we have $H^{p}\left(F_{n}\left(\mathbb{C}^{m}\right)\right)=0$ for $1 \leq p \leq 2 m-2$. Then the homomorphism $i^{*}$ : $H^{q}\left(\mathrm{Ch}_{n}(m)_{B}\right) \rightarrow H^{q}\left(Y_{C}\right)$ is an isomorphism for $q \leq 2 m-2$. Let $t_{j}$ be an element of $H^{2}\left(\mathrm{Ch}_{n}(m)_{B}\right)$ such that $i^{*}\left(t_{j}\right)=t_{j}^{\prime}$ for $j=1, \ldots, n-1$. Then we have $t_{j}{ }^{m-1}=0$ for $j=1, \ldots, n-1$.

We regard $H^{*}\left(\mathrm{Ch}_{n}(m)_{B}\right)$ as an algebra over $H^{*}\left(F_{n}\left(\mathbb{C}^{m}\right)\right)$ by using the ring homomorphism $\Psi_{n, m}^{*}: H^{*}\left(F_{n}\left(\mathbb{C}^{m}\right)\right) \rightarrow H^{*}\left(\mathrm{Ch}_{n}(m)_{B}\right)$.

Theorem 5.2. The cohomology ring of $\mathrm{Ch}_{n}(m)_{B}$ is a truncated polynomial algebra generated by $t_{j},(j=1, \ldots, n-1)$ over $H^{*}\left(F_{n}\left(\mathbb{C}^{m}\right)\right)$ :

$$
H^{*}\left(\mathrm{Ch}_{n}(m)_{B}\right) \cong H^{*}\left(F_{n}\left(\mathbb{C}^{m}\right)\right) \otimes \mathbb{Z}\left[t_{1}, \ldots, t_{n-1}\right] /\left(t_{1}^{m-1}, \ldots, t_{n-1}^{m-1}\right) .
$$

Proof. By the above argument, we have a ring homomorphism

$$
\psi: \mathbb{Z}\left[t_{1}, \ldots, t_{n-1}\right] /\left(t_{1}^{m-1}, \ldots, t_{n-1}^{m-1}\right) \longrightarrow H^{*}\left(\mathrm{Ch}_{n}(m)_{B}\right) .
$$

Then the ring homomorphism

$$
H^{*}\left(F_{n}\left(\mathbb{C}^{m}\right)\right) \otimes \mathbb{Z}\left[t_{1}, \ldots, t_{n-1}\right] /\left(t_{1}^{m-1}, \ldots, t_{n-1}^{m-1}\right) \stackrel{\Psi_{n, m}^{*} \otimes \psi}{\longrightarrow} H^{*}\left(\mathrm{Ch}_{n}(m)_{B}\right)
$$

gives an isomorphism.

\section{Cohomology of $\operatorname{Rep}_{n}(m)_{B}$.}

In $\S 3.4$ we described the scheme $\operatorname{Rep}_{n}(m)_{B}$ over $\mathbb{Z}$ as a fibre bundle over the flag scheme Flag $\left(\mathbb{A}_{\mathbb{Z}}^{n}\right)$. In the following we abbreviate the $\mathbb{C}$-valued points of $\operatorname{Rep}_{n}(m)_{B}$ with classical topology to $\operatorname{Rep}_{n}(m)_{B}$. In this section we consider the cohomology of $\operatorname{Rep}_{n}(m)_{B}$ for $m \geq 2$ by using the Serre spectral sequence associated with the fibre bundle.

First, we recall the cohomology ring of the flag manifold $U(n) / T^{n}$. We say that a sequence $\left(L_{1}, \ldots, L_{n-1}\right)$ of subvector spaces in $\mathbb{C}^{n}$ is a complete flag if $L_{i} \subset L_{i+1}$ for $i=1, \ldots, n-2$ and $\operatorname{dim}_{\mathbb{C}} L_{i}=i$ for $i=1, \ldots, n-$ 1. Let $\operatorname{Flag}\left(\mathbb{C}^{n}\right)$ be the set of all complete flags in the vector space $\mathbb{C}^{n}$. Then $\mathrm{PGL}_{n}(\mathbb{C})$ acts on Flag $\left(\mathbb{C}^{n}\right)$ transitively. Let $\mathbb{C}^{i}$ be the subspace of $\mathbb{C}^{n}$ spanned by the first $i$ canonical basis vectors for $i=1, \ldots, n-1$. Then we see that the stabilizer of the complete flag $\left(\mathbb{C}^{1}, \ldots, \mathbb{C}^{n-1}\right)$ is $\mathrm{B}_{n}(\mathbb{C})$. We regard Flag $\left(\mathbb{C}^{n}\right)$ as a manifold by means of the isomorphism $\operatorname{Flag}\left(\mathbb{C}^{n}\right) \cong$ $\mathrm{PGL}_{n}(\mathbb{C}) / \mathrm{B}_{n}(\mathbb{C})$. Let $U(n)$ be the unitary group of size $n$ and let $T^{n}$ be a maximal torus of $U(n)$ consisting of the diagonal matrices. Then $U(n)$ also acts on Flag $\left(\mathbb{C}^{n}\right)$ transitively and the stabilizer group of $\left(\mathbb{C}^{1}, \ldots, \mathbb{C}^{n-1}\right)$ is $T^{n}$. Hence we get an isomorphism $\operatorname{Flag}\left(\mathbb{C}^{n}\right) \cong U(n) / T^{n}$. Let $\pi_{i}: T^{n} \rightarrow T^{1}$ 
be the $i$ th projection for $i=1, \ldots, n$. Then we have a line bundle $E_{i}$ over $\operatorname{Flag}\left(\mathbb{C}^{n}\right)$ :

$$
U(n) \times_{\pi_{i}} \mathbb{C} \longrightarrow \operatorname{Flag}\left(\mathbb{C}^{n}\right) .
$$

We denote by $t_{i}$ the first Chern class of the line bundle $E_{i}$ :

$$
t_{i}=c_{1}\left(E_{i}\right) \in H^{2}\left(\operatorname{Flag}\left(\mathbb{C}^{n}\right)\right) .
$$

Then we have the following well-known lemma:

Lemma 6.1. The cohomology ring of $\operatorname{Flag}\left(\mathbb{C}^{n}\right)$ is given by

$$
H^{*}\left(\operatorname{Flag}\left(\mathbb{C}^{n}\right)\right)=\mathbb{Z}\left[t_{1}, \ldots, t_{n}\right] /\left(c_{1}, \ldots, c_{n}\right),
$$

where $c_{i}$ is the ith symmetric function for $i=1, \ldots, n$.

We note that $H^{i}\left(\operatorname{Flag}\left(\mathbb{C}^{n}\right)\right)=0$ for $i>n^{2}-n$ since $\operatorname{Flag}\left(\mathbb{C}^{n}\right)$ is a closed manifold of real dimension $n^{2}-n$.

The space $\operatorname{Rep}_{n}(m)_{B}$ is defined as $\mathrm{B}_{n}(m)_{B} \times_{\mathrm{B}_{n}(\mathbb{C})} \mathrm{PGL}_{n}(\mathbb{C})$. We note that there is an isomorphism $\operatorname{Rep}_{n}(m)_{B} \cong \mathrm{B}_{n}(m)_{B} \times_{T_{\mathbb{R}}} P U(n)$ where $P U(n)$ is the projective unitary group and $T_{\mathbb{R}}$ is its maximal torus. We define $\operatorname{Rep}_{n}(m)_{B}^{\prime}$ as $\mathrm{B}_{n}(m)_{B}^{\prime} \times_{T_{\mathbb{R}}} P U(n)$.

Lemma 6.2. There is a homotopy equivalence $\operatorname{Rep}_{n}(m)_{B} \simeq \operatorname{Rep}_{n}(m)_{B}^{\prime}$.

Proof. This follows from Lemma 4.4.

By Lemma 6.2, the natural map $\operatorname{Rep}_{n}(m)_{B}^{\prime} \rightarrow \operatorname{Rep}_{n}(m)_{B}$ induces an isomorphism of cohomology rings. Hence we calculate the cohomology of $\operatorname{Rep}_{n}(m)_{B}^{\prime}$. There is a fibre bundle

$$
\mathrm{B}_{n}(m)_{B}^{\prime} \longrightarrow \operatorname{Rep}_{n}(m)_{B}^{\prime} \longrightarrow \operatorname{Flag}\left(\mathbb{C}^{n}\right) .
$$

Then we obtain the associated Serre spectral sequence

$$
E_{2}^{p, q}=H^{p}\left(\operatorname{Flag}\left(\mathbb{C}^{n}\right) ; \mathcal{H}^{q}\left(\mathrm{~B}_{n}(m)_{B}^{\prime}\right)\right) \Longrightarrow H^{p+q}\left(\operatorname{Rep}_{n}(m)_{B}^{\prime}\right) .
$$

Since Flag $\left(\mathbb{C}^{n}\right)$ is simply connected, the coefficient system is trivial. By Theorem 4.3 and Lemma 6.1 , the cohomology group of $\mathrm{B}_{n}(m)_{B}^{\prime}$ and $\operatorname{Flag}\left(\mathbb{C}^{n}\right)$ are free over $\mathbb{Z}$. Hence we have an isomorphism

$$
E_{2}^{p, q} \cong H^{p}\left(\operatorname{Flag}\left(\mathbb{C}^{n}\right)\right) \otimes H^{q}\left(\mathrm{~B}_{n}(m)_{B}^{\prime}\right) .
$$

We recall that there is a map $\mathrm{B}_{n}(m)_{B}^{\prime} \rightarrow F_{n}\left(\mathbb{C}^{m}\right)$ which is a fibre bundle with fibre $Y_{B}^{\prime}$.

Lemma 6.3. Let $c \in H^{*}\left(\mathrm{~B}_{n}(m)_{B}^{\prime}\right)$. If $c$ is in the image of the homomorphism $H^{*}\left(F_{n}\left(\mathbb{C}^{m}\right)\right) \rightarrow H^{*}\left(\mathrm{~B}_{n}(m)_{B}^{\prime}\right)$, then $c$ is a permanent cycle.

Proof. This follows from the fact that there is a map $\operatorname{Rep}_{n}(m)_{B}^{\prime} \rightarrow F_{n}\left(\mathbb{C}^{m}\right)$ which factors through $\mathrm{B}_{n}(m)_{B}^{\prime} \rightarrow F_{n}\left(\mathbb{C}^{m}\right)$.

Corollary 6.4. The $E_{*}^{*, *}$ is a spectral sequence of $H^{*}\left(F_{n}\left(\mathbb{C}^{m}\right)\right)$-modules. 
Proposition 6.5. If $m>\left(n^{2}-n\right) / 2+1$, then the spectral sequence collapses from $E_{2}$-term. In this case we have

$$
H^{*}\left(\operatorname{Rep}_{n}(m)_{B}^{\prime}\right) \cong H^{*}\left(F_{n}\left(\mathbb{C}^{m}\right)\right) \otimes H^{*}\left(\operatorname{Flag}\left(\mathbb{C}^{n}\right)\right) \otimes \Lambda\left(s_{1}, \ldots, s_{n-1}\right)
$$

as algebras where the degree of $s_{i}$ is $2 m-3$ for $i=1, \ldots, n-1$.

Proof. Since $\mathrm{B}_{n}(m)_{B}^{\prime}$ is $(2 m-4)$-connected, we have $d_{2}=\cdots=d_{2 m-3}=0$. Then the proposition follows from the fact that $H^{i}\left(\operatorname{Flag}\left(\mathbb{C}^{n}\right)\right)=0$ for $i>$ $n^{2}-n$.

The first nontrivial differential $d_{2 m-2}$ is given by

$$
d_{2 m-2}\left(s_{i}\right)=\left(t_{i}-t_{i+1}\right)^{m-1} \text { for } 1 \leq i<n .
$$

Let $C$ be a differential graded algebra given by

$$
C=\mathbb{Z}\left[t_{1}, \ldots, t_{n}\right] /\left(c_{1}, \ldots, c_{n}\right) \otimes \Lambda\left(s_{1}, \ldots, s_{n-1}\right),
$$

where the cohomological degree of $t_{i}$ is 0 for $i=1, \ldots, n$ and the cohomological degree of $s_{i}$ is 1 for $i=1, \ldots, n-1$. The differential is defined by

$$
d\left(s_{i}\right)=\left(t_{i}-t_{i+1}\right)^{m-1}, \quad i=1, \ldots, n-1 .
$$

We denote by $H(C)$ the cohomology algebra of $C$.

Lemma 6.6. The $E_{2 m-1}$-term of the Serre spectral sequence of the fibre bundle $\mathrm{B}_{n}(m)_{B}^{\prime} \rightarrow \operatorname{Rep}_{n}(m)_{B}^{\prime} \rightarrow F_{n}\left(\mathbb{C}^{m}\right)$ is $H(C) \otimes H^{*}\left(F_{n}\left(\mathbb{C}^{m}\right)\right)$.

In the rest of this section we calculate the cohomology of $\operatorname{Rep}_{n}(m)$ for small $n$.

6.1. The case $\boldsymbol{n}=\mathbf{2}$. If $n=2$, the flag manifold Flag $\left(\mathbb{C}^{2}\right)$ is the 2 sphere $S^{2}$ and $P U(2)$ is the real projective space $\mathbb{R P}^{3}$. We recall that $\operatorname{Rep}_{2}(2)_{B}^{\prime}=\mathrm{B}_{2}(2)_{B}^{\prime} \times_{T_{\mathbb{R}}} P U(2)$. It is easy to see that the action of $T_{\mathbb{R}}$ is free and the quotient map $\mathrm{B}_{2}(2)_{B}^{\prime} \rightarrow \mathrm{B}_{2}(2)_{B}^{\prime} / T_{\mathbb{R}}$ is identified with the fibre bundle $\mathrm{B}_{2}(2)_{B}^{\prime} \rightarrow F_{2}\left(\mathbb{C}^{2}\right)$. Hence $\mathrm{B}_{2}(2)_{B}^{\prime} \rightarrow F_{2}\left(\mathbb{C}^{2}\right)$ is a principal $T_{\mathbb{R}}$-bundle. Since $T_{\mathbb{R}} \cong S^{1}$ and $F_{2}\left(\mathbb{C}^{2}\right)$ is 2-connected, the principal bundle is trivial and $\mathrm{B}_{2}(2)_{B}^{\prime} \cong F_{2}\left(\mathbb{C}^{2}\right) \times T_{\mathbb{R}}$. This implies that $\operatorname{Rep}_{2}(2)_{B}^{\prime} \cong F_{2}\left(\mathbb{C}^{2}\right) \times P U(2)$. It is also easy to construct an isomorphism explicitly.

Proposition 6.7. If $n=2$ and $m=2$, we have a homotopy equivalence $\operatorname{Rep}_{2}(2)_{B} \simeq F_{2}\left(\mathbb{C}^{2}\right) \times \mathbb{R} \mathbb{P}^{3}$. Hence its cohomology ring is given by

$$
H^{*}\left(\operatorname{Rep}_{2}(2)_{B}\right) \cong H^{*}\left(F_{2}\left(\mathbb{C}^{2}\right)\right) \otimes H^{*}\left(\mathbb{R P}^{3}\right) .
$$

If $n=2$ and $m \geq 3$, we have

$$
H^{*}\left(\operatorname{Rep}_{2}(m)_{B}\right) \cong H^{*}\left(F_{2}\left(\mathbb{C}^{m}\right)\right) \otimes H^{*}\left(\operatorname{Flag}\left(\mathbb{C}^{2}\right)\right) \otimes \Lambda(s)
$$

where $|s|=2 m-3$. 
Proof. The case $m=2$ follows from Lemma 6.2. The case $m=3$ follows from Proposition 6.5.

Remark 6.8. There exists a unique $\Upsilon_{m}$-invariant sub-line bundle $\mathcal{L}_{m}$ of $\mathcal{O}_{\operatorname{Rep}_{2}(m)_{B}}^{\oplus 2}$ on $\operatorname{Rep}_{2}(m)_{B}$. The line bundle $\mathcal{L}_{m}$ is obtained by the pull back of $\mathcal{E}_{1}$ of the universal flag by $\operatorname{Rep}_{2}(m)_{B} \rightarrow \operatorname{Flag}\left(\mathbb{C}^{2}\right)$ in $\S 3.4$. In the case $m=2$, we see that $0 \neq c_{1}\left(\mathcal{L}_{2}\right) \in H^{2}\left(\operatorname{Rep}_{2}(2)_{B}\right) \cong \mathbb{Z} / 2 \mathbb{Z}$ by [Na2] Proposition 4.5. We also see that $\mathcal{L}_{2}^{\otimes 2} \cong \mathcal{O}_{\operatorname{Rep}_{2}(2)_{B}}$ by [Na2] Proposition 4.7. In the case $m \geq 3$, we have $c_{1}\left(\mathcal{L}_{m}\right)=t_{1} \in H^{2}\left(\operatorname{Rep}_{2}(m)_{B}\right)=H^{2}\left(\operatorname{Flag}\left(\mathbb{C}^{2}\right)\right) \cong \mathbb{Z}$.

6.2. The case $\boldsymbol{n}=\mathbf{3}$. By Lemma 6.6 , the $E_{2 m-1}$-term of the Serre spectral sequence of the fibre bundle $\mathrm{B}_{3}(m)_{B}^{\prime} \rightarrow \operatorname{Rep}_{3}(m)_{B}^{\prime} \rightarrow$ Flag $\left(\mathbb{C}^{3}\right)$ is given by

$$
E_{2 m-1} \cong H(C) \otimes H^{*}\left(F_{3}\left(\mathbb{C}^{m}\right)\right) \text {. }
$$

Then the next nontrivial differential is $d_{4 m-5}$. Since $H^{*}\left(\operatorname{Flag}\left(\mathbb{C}^{3}\right)\right)$ is concentrated in even degrees, we see that $d_{4 m-5}(H(C))=0$. Hence we obtain the following proposition:

Proposition 6.9. If $n=3$, then we have $H^{*}\left(\operatorname{Rep}_{3}(m)_{B} ; k\right) \cong H^{*}(C \otimes k) \otimes$ $H^{*}\left(F_{3}\left(\mathbb{C}^{m}\right) ; k\right)$ as $H^{*}\left(F_{3}\left(\mathbb{C}^{m}\right) ; k\right)$-modules for any field $k$.

\section{Virtual Hodge polynomial.}

For every algebraic scheme over $\mathbb{C}$ we can define its virtual Hodge polynomial by virtue of Deligne's mixed Hodge theory ([De1] and [De2]). In this section, we calculate the virtual Hodge polynomials of the algebraic varieties $\mathrm{B}_{n}(m)_{B}, \operatorname{Ch}_{n}(m)_{B}$, and $\operatorname{Rep}_{n}(m)_{B}$ over $\mathbb{C}$. By these calculations we can determine the virtual Poincaré polynomials of the moduli of absolutely irreducible representations of degree 2 for free monoids (See $[\mathrm{Na} 3]$ ).

7.1. Definition of virtual Hodge polynomial. In this subsection, we give a survey on virtual Hodge polynomials. More precisely, see [DK], $[\mathrm{Ch} 1],[\mathrm{Ch} 2]$ and so on.

For an algebraic scheme $X$ over $\mathbb{C}$, we can define the virtual Hodge polynomial $H(X ; x, y)$ of $X$ in $\mathbb{Z}[x, y]$ which satisfies the following properties:

(1) For a smooth projective variety $X$ over $\mathbb{C}$,

$$
H(X ; x, y)=\sum_{p, q} h^{p, q}(X) x^{p} y^{q},
$$

where $h^{p, q}(X)$ is the $(p, q)$-th Hodge number of $X$.

(2) Let $U$ be a Zariski open subset of $X$. Set $Z:=X \backslash U$. Then we have

$$
H(X ; x, y)=H(U ; x, y)+H(Z ; x, y) \text {. }
$$

(3) Let $f: E \rightarrow B$ be a fibre bundle with fibre $F$ which has a local trivialization with respect to Zariski topology. Then we have

$$
H(E ; x, y)=H(B ; x, y) H(F ; x, y) \text {. }
$$


(4) For a bijective morphism $f: X \rightarrow Y, H(X ; x, y)=H(Y ; x, y)$.

Remark 7.1. For the virtual Hodge polynomial $H(X ; x, y)$ of an algebraic scheme $X$ over $\mathbb{C}$, we call the polynomial $H(X ; t, t) \in \mathbb{Z}[t]$ the virtual Poincaré polynomial of $X$. See also $[\mathbf{F u}] \S 4.5$ for virtual Poincaré polynomials.

Example 7.2. By the above properties we easily obtain

$$
\begin{aligned}
& H\left(\mathbb{P}^{n} ; x, y\right)=1+x y+x^{2} y^{2}+\cdots+x^{n} y^{n}, \\
& H\left(\mathbb{C}^{n} ; x, y\right)=x^{n} y^{n} .
\end{aligned}
$$

Notation 7.3. In the sequel, we put $z=x y$.

Let us calculate several virtual Hodge polynomials.

Example 7.4. The virtual Hodge polynomial of $\mathrm{GL}_{n}(\mathbb{C})$ can be calculated as follows: The group $\mathrm{GL}_{n}(\mathbb{C})$ acts on $\mathbb{P}^{n-1}$ canonically. The stabilizer of $(1: 0: \cdots: 0)$ is isomorphic to $\mathrm{GL}_{n-1}(\mathbb{C}) \times \mathrm{GL}_{1}(\mathbb{C}) \times \mathbb{C}^{n-1}$ as an algebraic scheme. By considering the fibre bundle $\mathrm{GL}_{n-1}(\mathbb{C}) \times \mathrm{GL}_{1}(\mathbb{C}) \times \mathbb{C}^{n-1} \rightarrow$ $\mathrm{GL}_{n}(\mathbb{C}) \rightarrow \mathbb{P}^{n-1}$, we have

$$
H\left(\mathrm{GL}_{n}(\mathbb{C})\right)=H\left(\mathrm{GL}_{n-1}(\mathbb{C})\right) H\left(\mathrm{GL}_{1}(\mathbb{C})\right) H\left(\mathbb{C}^{n-1}\right) H\left(\mathbb{P}^{n-1}\right) .
$$

Since $H\left(\mathrm{GL}_{1}(\mathbb{C})\right)=z-1$, we obtain

$$
H\left(\mathrm{GL}_{n}(\mathbb{C})\right)=z^{(n-1) n / 2} \prod_{k=1}^{n}\left(z^{k}-1\right)
$$

by induction. We also have

$$
H\left(\mathrm{PGL}_{n}(\mathbb{C})\right)=z^{(n-1) n / 2} \prod_{k=2}^{n}\left(z^{k}-1\right)
$$

by the fibre bundle $\mathrm{GL}_{1}(\mathbb{C}) \rightarrow \mathrm{GL}_{n}(\mathbb{C}) \rightarrow \mathrm{PGL}_{n}(\mathbb{C})$.

Let $X$ be an algebraic scheme over $\mathbb{C}$. We calculate the virtual Hodge polynomial of the configuration space $F_{n}(X)$ of $X$. The following proposition has been proved in $[\mathbf{F M}]$ Proposition 2.1, essentially.

Proposition 7.5. Let $H(X)$ be the virtual Hodge polynomial of $X$. Then the virtual Hodge polynomial $H\left(F_{n}(X)\right)$ of $F_{n}(X)$ is given by

$$
H\left(F_{n}(X)\right)=\prod_{k=0}^{n-1}(H(X)-k) .
$$

Proof. We prove the statement by induction on $n$. If $n=1$, then it is obvious since $F_{1}(X)=X$. Suppose that the statement is true until $n-1$. The scheme $X \times F_{n-1}(X)$ is a disjoint union of $F_{n}(X)$ and $(n-1)$ pieces of subschemes 
which are isomorphic to $F_{n-1}(X)$. Hence we have $H(X) H\left(F_{n-1}(X)\right)=$ $H\left(F_{n}(X)\right)+(n-1) H\left(F_{n-1}(X)\right)$, which easily follows the statement.

As a corollary, we have:

Corollary 7.6. The virtual Hodge polynomial $H\left(F_{n}\left(\mathbb{C}^{m}\right)\right)$ of the configuration space $F_{n}\left(\mathbb{C}^{m}\right)$ is given by

$$
H\left(F_{n}\left(\mathbb{C}^{m}\right)\right)=\prod_{k=0}^{n-1}\left(z^{m}-k\right) .
$$

\subsection{The virtual Hodge polynomial of the moduli of representa-} tions with Borel mold. In this subsection, we calculate the virtual Hodge polynomials of $\mathrm{B}_{n}(m)_{B}, \mathrm{Ch}_{n}(m)_{B}$, and $\operatorname{Rep}_{n}(m)_{B}$.

By Proposition 3.6 we see that $\mathrm{B}_{n}(m)_{B} \rightarrow F_{n}\left(\mathbb{C}^{m}\right)$ is a fibre bundle with fibre $\left(\mathbb{C}^{m} \backslash \mathbb{C}^{1}\right)^{n-1} \times\left(\mathbb{C}^{m}\right)^{(n-1)(n-1) / 2}$ over $\mathbb{C}$. Hence we have:

Proposition 7.7. The virtual Hodge polynomial $H\left(\mathrm{~B}_{n}(m)_{B}\right)$ of $\mathrm{B}_{n}(m)_{B}$ is given by

$$
H\left(\mathrm{~B}_{n}(m)_{B}\right)=\left(z^{m}-z\right)^{n-1} z^{m(n-2)(n-1) / 2} \prod_{k=0}^{n-1}\left(z^{m}-k\right) .
$$

Proposition 3.8 follows that $\mathrm{Ch}_{n}(m)_{B} \rightarrow F_{n}\left(\mathbb{C}^{m}\right)$ is a fibre bundle with fibre $\mathbb{P}^{m-2} \times\left(\mathbb{C}^{m-1}\right)^{(n-2)(n-1) / 2}$ over $\mathbb{C}$. We also see that $\mathrm{B}_{n}(m)_{B} \rightarrow \mathrm{Ch}_{n}(m)_{B}$ is a $\mathrm{B}_{n}(\mathbb{C})$-principal fibre bundle with respect to Zariski topology. From these facts we have:

Proposition 7.8. The virtual Hodge polynomial $H\left(\mathrm{Ch}_{n}(m)_{B}\right)$ of $\mathrm{Ch}_{n}(m)_{B}$ is given by

$$
H\left(\mathrm{Ch}_{n}(m)_{B}\right)=\frac{\left(z^{m-1}-1\right)^{n-1}}{(z-1)^{n-1}} z^{(m-1)(n-2)(n-1) / 2} \prod_{k=0}^{n-1}\left(z^{m}-k\right) .
$$

By the fact that $\operatorname{Rep}_{n}(m)_{B} \rightarrow \mathrm{Ch}_{n}(m)_{B}$ is a $\mathrm{PGL}_{n}(\mathbb{C})$-principal fibre bundle with respect to Zariski topology, we can calculate $H\left(\operatorname{Rep}_{n}(m)_{B}\right)$ as follows:

Proposition 7.9. The virtual Hodge polynomial $H\left(\operatorname{Rep}_{n}(m)_{B}\right)$ is given by

$$
H\left(\operatorname{Rep}_{n}(m)_{B}\right)=\frac{\left(z^{m}-z\right)^{n-1}}{(z-1)^{n-1}} z^{m(n-2)(n-1) / 2} \prod_{k=0}^{n-1}\left(z^{m}-k\right) \prod_{k=2}^{n}\left(z^{k}-1\right) .
$$




\section{Remarks on the case $\boldsymbol{m}=\infty$.}

There is a natural inclusion map $\mathrm{B}_{n}(m)_{B} \hookrightarrow \mathrm{B}_{n}(m+1)_{B}$ given by $\left(A_{1}, \ldots\right.$, $\left.A_{m}\right) \mapsto\left(A_{1}, \ldots, A_{m}, 0\right)$. Then we see that this map also induces natural inclusion maps $\mathrm{Ch}_{n}(m)_{B} \hookrightarrow \mathrm{Ch}_{n}(m+1)_{B}$ and $\operatorname{Rep}_{n}(m)_{B} \hookrightarrow \operatorname{Rep}_{n}(m+1)_{B}$. We define the spaces $\mathrm{B}_{n}(\infty)_{B}, \mathrm{Ch}_{n}(\infty)_{B}$ and $\operatorname{Rep}_{n}(\infty)_{B}$ to be the homotopy direct limits (telescopes) of the following systems, respectively:

$$
\begin{aligned}
& \mathrm{B}_{n}(2)_{B} \hookrightarrow \mathrm{B}_{n}(3)_{B} \hookrightarrow \cdots \quad \cdots \quad \mathrm{B}_{n}(m)_{B} \hookrightarrow \cdots \\
& \mathrm{Ch}_{n}(2)_{B} \hookrightarrow \mathrm{Ch}_{n}(3)_{B} \hookrightarrow \quad \cdots \quad \hookrightarrow \quad \mathrm{Ch}_{n}(m)_{B} \quad \hookrightarrow \cdots \\
& \operatorname{Rep}_{n}(2)_{B} \hookrightarrow \operatorname{Rep}_{n}(3)_{B} \hookrightarrow \cdots \hookrightarrow \operatorname{Rep}_{n}(m)_{B} \hookrightarrow \cdots .
\end{aligned}
$$

In this section we study $\mathrm{B}_{n}(\infty)_{B}, \mathrm{Ch}_{n}(\infty)_{B}$ and $\operatorname{Rep}_{n}(\infty)_{B}$.

The inclusion $\mathbb{C}^{m} \hookrightarrow \mathbb{C}^{m+1}$ given by $\left(z_{1}, \ldots, z_{m}\right) \mapsto\left(z_{1}, \ldots, z_{m}, 0\right)$ defines an inclusion $F_{n}\left(\mathbb{C}^{m}\right) \hookrightarrow F_{n}\left(\mathbb{C}^{m+1}\right)$. We denote by $F_{n}\left(\mathbb{C}^{\infty}\right)$ the homotopy direct limit of the system

$$
F_{n}\left(\mathbb{C}^{2}\right) \hookrightarrow F_{n}\left(\mathbb{C}^{3}\right) \hookrightarrow \cdots \hookrightarrow F_{n}\left(\mathbb{C}^{m}\right) \hookrightarrow \cdots .
$$

The following lemma follows from Lemma 4.2 and the fact that the configuration space $F_{n}\left(\mathbb{C}^{m}\right)$ is $(2 m-2)$-connected.

Lemma 8.1. $F_{n}\left(\mathbb{C}^{\infty}\right)$ and $\mathrm{B}_{n}(\infty)_{B}$ are weakly contractible.

We recall that there is a fibre bundle

$$
\left(\mathbb{C P}^{m-2}\right)^{n-1} \longrightarrow \mathrm{Ch}_{n}(m)_{B}^{\prime} \longrightarrow F_{n}\left(\mathbb{C}^{m}\right) \text {. }
$$

By the long exact sequence of homotopy groups associated with the fibre bundle, $\left(\mathbb{C P}^{m-2}\right)^{n-1} \rightarrow \mathrm{Ch}_{n}(m)_{B}^{\prime}$ induces isomorphisms of homotopy groups up to dimension $2 m-3$. There is a commutative diagram

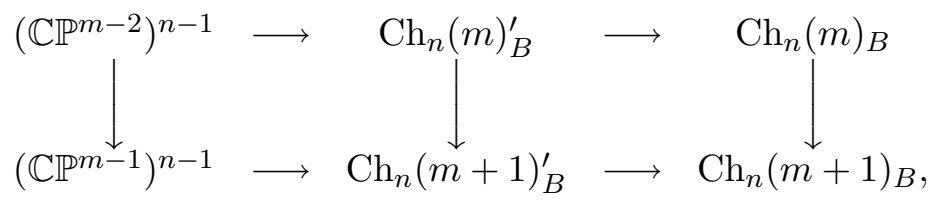

where the vertical arrows are natural inclusions. This diagram induces a map

$$
\left(\mathbb{C P}^{\infty}\right)^{n-1} \longrightarrow \mathrm{Ch}_{n}(\infty)_{B}^{\prime} \longrightarrow \mathrm{Ch}_{n}(\infty)_{B}
$$

Proposition 8.2. $\left(\mathbb{C P}^{\infty}\right)^{n-1} \rightarrow \mathrm{Ch}_{n}(\infty)_{B}$ is a weak homotopy equivalence.

Proof. Since $\left(\mathbb{C P}^{m-2}\right)^{n-1} \rightarrow \mathrm{Ch}_{n}(m)_{B}^{\prime}$ is a homotopy equivalence up to dimension $2 m-3$, the map $\left(\mathbb{C P}^{\infty}\right)^{n-1} \rightarrow \mathrm{Ch}_{n}(\infty)_{B}^{\prime}$ is a weak homotopy equivalence. The homotopy equivalence $\mathrm{Ch}_{n}(m)_{B}^{\prime} \hookrightarrow \mathrm{Ch}_{n}(m)_{B}$ implies that $\mathrm{Ch}_{n}(\infty)_{B}^{\prime} \rightarrow \mathrm{Ch}_{n}(\infty)_{B}$ is a weak homotopy equivalence. 
The homotopy direct limit of the fibre bundles $T_{\mathbb{R}} \rightarrow \mathrm{B}_{n}(m)_{B}^{\prime} \rightarrow \mathrm{Ch}_{n}(m)_{B}^{\prime}$ is a model of universal principal $T_{\mathbb{R}}$-bundle.

Corollary 8.3. The cohomology of $\mathrm{Ch}_{n}(\infty)_{B}$ is given by

$$
H^{*}\left(\mathrm{Ch}_{n}(\infty)_{B}\right) \cong \mathbb{Z}\left[t_{1}, \ldots, t_{n-1}\right],
$$

where the degree of $t_{i}$ is 2 for $i=1, \ldots, n-1$.

We recall that there is a map from $\operatorname{Rep}_{n}(m)_{B}$ to $\operatorname{Flag}\left(\mathbb{C}^{n}\right)$ which is compatible with the inclusions $\operatorname{Rep}_{n}(m)_{B} \hookrightarrow \operatorname{Rep}_{n}(m+1)_{B}$. Hence we obtain a map $\operatorname{Rep}_{n}(\infty)_{B} \rightarrow \operatorname{Flag}\left(\mathbb{C}^{n}\right)$.

Proposition 8.4. $\operatorname{Rep}_{n}(\infty)_{B} \rightarrow \mathrm{Flag}\left(\mathbb{C}^{n}\right)$ is a weak homotopy equivalence. Hence the cohomology ring of $\operatorname{Rep}_{n}(\infty)_{B}$ is given by

$$
H^{*}\left(\operatorname{Rep}_{n}(\infty)_{B}\right) \cong \mathbb{Z}\left[t_{1}, \ldots, t_{n}\right] /\left(c_{1}, \ldots, c_{n}\right) .
$$

Proof. The fibre of $\operatorname{Rep}_{n}(m)_{B} \rightarrow \operatorname{Flag}\left(\mathbb{C}^{n}\right)$ is $\mathrm{B}_{n}(m)_{B}$. Then the proposition follows from Lemma 8.1.

\section{References}

[Ch1] J. Cheah, On the cohomology of Hilbert schemes of points, Jour. Alg. Geom., 5 (1996), 479-511, MR 97b:14005, Zbl 0889.14001.

[Ch2] - The virtual Hodge polynomials of nested Hilbert schemes and related varieties, Math. Z., 227 (1998), 479-504, MR 2000c:14007, Zbl 0890.14003.

[Co1] F.R. Cohen, The homology of $C_{n+1}$-spaces, $n \geq 0$, in 'The Homology of Iterated Loop Spaces' (F.R. Cohen, T.J. Lada and J.P. May), Lecture Notes in Mathematics, 533, Springer-Verlag, Berlin-New York, 1976, 207-351, MR 55 \#9096, Zbl 0334.55009.

[Co2] On configuration spaces, their homology, and Lie algebras, J. Pure Appl. Algebra, 100 (1995), 19-42, MR 96d:55005, Zbl 0921.57011.

[DK] V.I. Danilov and A.G. Khovanskiǔ, Newton polyhedra and an algorithm for computing Hodge-Deligne numbers, Math. USSR-Izv., 29 (1987), 279-298, MR 88i:32032, Zbl 0669.14012.

[De1] P. Deligne, Théorie de Hodge, II, Inst. Hautes Études Sci. Publ. Math., 40 (1971), 5-57, MR 58 \#16653a, Zbl 0219.14007.

[De2] _ Théorie de Hodge, III, Inst. Hautes Études Sci. Publ. Math., 44 (1974), 5-77, MR 58 \#16653b, Zbl 0237.14003.

[Fu] W. Fulton, Introduction to Toric Varieties, Annals of Mathematics Studies, 131, Princeton University Press, 1993, MR 94g:14028, Zbl 0813.14039.

[FM] W. Fulton and R. MacPherson, A compactification of configuration spaces, Ann. of Math., 139(2) (1994), 183-225, MR 95j:14002, Zbl 0820.14037.

[Na1] K. Nakamoto, Representation varieties and character varieties, Publ. Res. Inst. Math. Sci., 36(2) (2000), 159-189, MR 2001f:14026.

[Na2] - The moduli of representations with Borel mold, in preparation. 
[Na3] _ The moduli of representations of degree 2, in preparation.

Received May 15, 2002. T. Torii was partially supported by JSPS Research Fellowships for Young Scientists.

Department of General Education

Takuma National College of Technology

KAGAWA 769-1192

JAPAN

E-mail address: nakamoto@dg.takuma-ct.ac.jp

Department of Applied Mathematics

FUKUOKA UNIVERSITY

FUKUOKA 814-0180

JAPAN

E-mail address: torii@bach.sm.fukuoka-u.ac.jp 